\title{
Gut
}

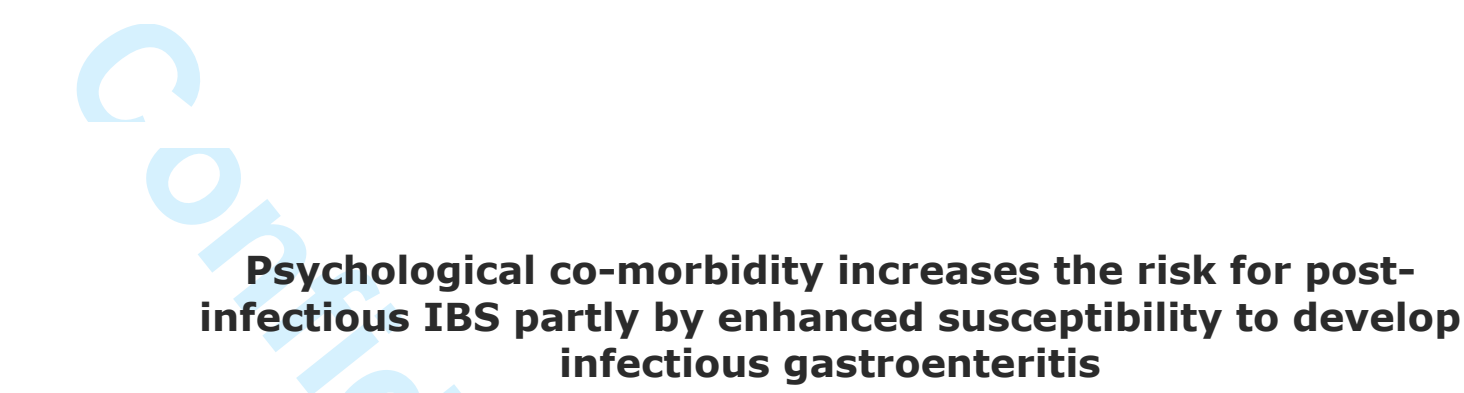

\begin{tabular}{|c|c|}
\hline Journal: & Gut \\
\hline Manuscript ID: & gutjnl-2015-309460.R2 \\
\hline Article Type: & Original Article \\
\hline Date Submitted by the Author: & $n / a$ \\
\hline Complete List of Authors: & $\begin{array}{l}\text { Wouters, Mira; University Hospital Leuven, KULeuven, Translational } \\
\text { Research Center for Gastrointestinal Disorders (TARGID) } \\
\text { Van Wanrooy, Sander; University Hospital Leuven, KULeuven, Translational } \\
\text { Research Center for Gastrointestinal Disorders (TARGID) } \\
\text { Ngyen, Anh; Leuven University, Department of Microbiology and } \\
\text { Immunology; VIB Leuven, Autoimmune Genetics Laboratory } \\
\text { Dooley, James; Leuven University, Department of Microbiology and } \\
\text { Immunology; VIB Leuven, Autoimmune Genetics Laboratory } \\
\text { Aguilera, Javier; University Hospital Leuven, KULeuven, Translational } \\
\text { Research Center for Gastrointestinal Disorders (TARGID) } \\
\text { Van Brabant, Winde; University Hospital Leuven, KULeuven, Translational } \\
\text { Research Center for Gastrointestinal Disorders (TARGID) } \\
\text { Garcia-Perez, Josselyn; Leuven University, Department of Microbiology and } \\
\text { Immunology; VIB Leuven, Autoimmune Genetics Laboratory } \\
\text { Van Oudenhove, Lukas; University Hospital Leuven, KULeuven, } \\
\text { Translational Research Center for Gastrointestinal Disorders (TARGID) } \\
\text { Van Ranst, Marc; Leuven University, Laboratory of Clinical Virology, Rega } \\
\text { Institute for Medical Research } \\
\text { Verhaegen, Jan; Leuven University, Department of Microbiology } \\
\text { Liston, Adrian; Leuven University, Department of Microbiology and } \\
\text { Immunology; VIB Leuven, Autoimmune Genetics Laboratory } \\
\text { Boeckxstaens, Guy; University Hospital Leuven, KULeuven, Translational } \\
\text { Research Center for Gastrointestinal Disorders (TARGID) }\end{array}$ \\
\hline Keywords: & IGE, IRRITABLE BOWEL SYNDROME \\
\hline
\end{tabular}




\section{Psychological co-morbidity increases the risk for post-infectious IBS partly by enhanced susceptibility to develop infectious gastroenteritis}

Mira Wouters 1\&, Sander Van Wanrooy 1\&, Anh Nguyen 2,3\&, James Dooley 2,3, Javier AguileraLizarraga 1, Winde Van Brabant 1, Josselyn E Garcia-Perez 2,3, Lukas Van Oudenhove 1, Marc Van Ranst 4, Jan Verhaegen 5, Adrian Liston 2,3*, Guy Boeckxstaens 1*

\& MW, SVW and AN should be considered as first authors with equal contribution.

* AL and GB share senior authorship.

1 Translational Research Center for Gastrointestinal Disorders, Dept. of Clinical and Experimental Medicine, University Hospital Leuven, KU Leuven, Leuven, Belgium

2 Department of Microbiology and Immunology, KU Leuven, Leuven, Belgium

3 Autoimmune Genetics Laboratory, VIB, Leuven, Belgium

4 Laboratory of Clinical Virology, Rega Institute for Medical Research, Catholic University of Leuven, Leuven, Belgium

5 Department of Microbiology, University Hospital Leuven, KU Leuven, Leuven, Belgium

Correspondence address:

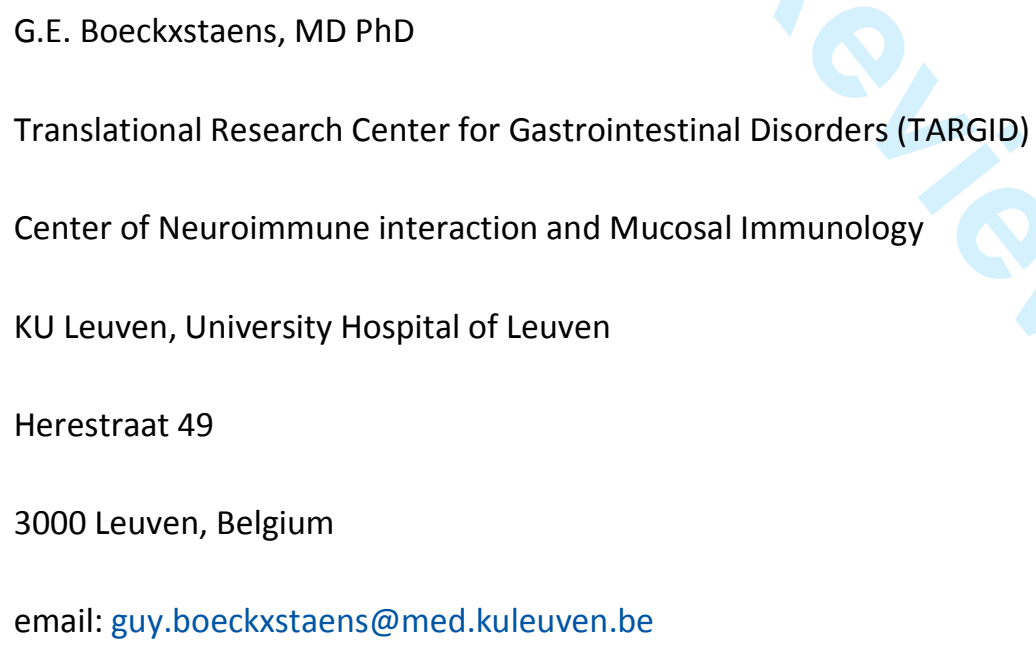

Key words: post-infectious irritable bowel syndrome, PI-IBS, Th2, anxiety 
Word count: 4708

The Corresponding Author has the right to grant on behalf of all authors and does grant on behalf of all authors, an exclusive licence (or non exclusive for government employees) on a worldwide basis to the BMJ Publishing Group Ltd and its Licensees to permit this article (if accepted) to be published in Gut editions and any other BMJPGL products to exploit all subsidiary rights, as set out in our licence 


\section{ABSTRACT:}

Objective: Psychological factors increase the risk to develop post-infectious irritable bowel syndrome (PI-IBS), but the mechanisms involved are unclear. As stress affects the immune system, we investigated the potential interaction between psychological factors, the immune response against infectious gastroenteritis (IGE) and the development of IGE and PI-IBS in a large cohort exposed to contaminated drinking water.

Design: 18620 people exposed to contaminated drinking water (Norovirus, Giardia lamblia, Campylobacter jejuni) were invited to participate in a prospective controlled cohort study. They were asked to complete questionnaires assessing demographic, psychological and clinical data during the outbreak and one year later. At both time points, in-depth immune function (peripheral blood and rectal biopsies) was studied in a subgroup of subjects.

Results: 1379 subjects completed the questionnaires during the outbreak, of which 271 developed IGE. Risk factors for IGE included younger age, pre-existing dyspepsia-like symptoms, anxiety and drinking contaminated tap water. Anxiety scores before the outbreak inversely correlated with IL2expressing CD4+Tcells ( $r=0.6, p=0.01, n=23)$. At follow-up, 34 of $172(20 \%)$ IGE subjects developed IBS compared to $24 / 366$ exposed participants (7\%, $p<0.0001, \chi 2$ test). A Th2 cytokine phenotype at time of infection was associated with increased risk for PI-IBS 1 year later. Except for increased B cell numbers, no evidence for systemic or rectal mucosal immune activation in PI-IBS was demonstrated at follow-up.

Conclusion: Our study shows that the increased risk of patients with psychological co-morbidity to develop PI-IBS may partly result from an increased susceptibility to develop IGE, possibly resulting from a Th2-immune bias. 


\section{SUMMARY BOX:}

What is already known about this subject:

- Up to $30 \%$ of individuals develop PI-IBS following infectious gastroenteritis

- Psychological factors increase the susceptibility to develop PI-IBS

- Psychological stress alters the T helper 1 and T helper 2 (Th1/Th2) immune balance

\section{What are the new findings:}

- Anxiety and somatization are risk factors to develop IGE

- Psychological factors, in particular anxiety and somatization, increase the risk to develop PIIBS partly by a direct effect and partly indirect via increased susceptibility to develop IGE

- Anxiety and depression at the time of infection may alter the Th1/Th2 immune balance, predisposing to PI-IBS

- Except for increased B cell numbers, there was no evidence for persistent immune activation in blood or rectal biopsies of PI-IBS

\section{How might it impact on clinical practice in the foreseeable future?}

- Our results provide further insight in the pathogenesis of PI-IBS, underscoring the impact of psychological factors

- The absence of inflammation in PI-IBS suggests that anti-inflammatory treatment with agents such as mesalazine are unlikely to be effective 


\section{INTRODUCTION:}

Acute infectious gastroenteritis (IGE) is a well-defined risk factor to develop irritable bowel syndrome (IBS), with $8-31 \%$ of patients developing post-infectious IBS (PI-IBS) (1-10). To date, persistent mucosal microscopic inflammation is proposed to underlie abnormal neuromuscular function contributing to altered bowel habits and increased abdominal pain (11). Increased number of mononuclear cells and higher levels of IL1 $\beta$ mRNA have indeed been reported in rectal biopsies collected 3 months to 1 year after IGE (12;13). In line, 3 months after a Campylobacter infection, Dunlop et al. (2) showed a small increase in enterochromaffin cells in PI-IBS patients compared to infected subjects who did not develop IBS. However, the number of lamina propria T cells was higher in patients with PI-IBS and post-infected controls compared to healthy volunteers. Moreover, no differences in T lymphocytes, mast cells or pro-inflammatory cytokines were detected between PI-IBS patients and infected control subjects three years after a Salmonella infection, although only PI-IBS patients were hypersensitive to rectal distention (14). Hence, the evidence supporting the hypothesis that chronic microscopic inflammation or immune activation is involved in PI-IBS is rather limited, confined to small patient numbers and mostly based on biopsies collected 3-4 months after the episode of IGE, and thus clearly deserves further study. Of note, the severity of the insult to the mucosa differs significantly between pathogens, most likely explaining why some studies still report inflammatory changes after 3-6 months and others do not. For example, Campylobacter jejuni produces a severe insult with mucosal ulceration while viruses rather produce a more transient inflammation without ulcerations. The risk to develop PI-IBS is not only determined by the nature of the pathogen, the severity of the infection, age and gender, but also by psychological factors (15). Anxiety, depression and fatigue are identified as risk factors for PI-IBS $(16 ; 17)$. Correspondingly, patients with a history of anxiety and depression have impaired recovery of PI-IBS during a six to eight year period of follow-up $(17 ; 18)$. How psychological factors may increase the risk to develop PIIBS however remains unclear. Of interest, it is becoming increasingly clear that brain-gut interactions may significantly modulate the intestinal immune system, and thus the immune response to a pathogen. Chronic psychological stress impairs the immune system by altering patterns of cytokine secretion, thereby increasing the vulnerability to infections $(19 ; 20)$. For example, chronic psychological stress in rats alters the immune response from antigen-induced oral tolerance to sensitization with a subsequent antigen-induced increase in inflammatory cells and mucosal permeability (21). Likewise, chronic stress alters the Th1/Th2 balance $(21 ; 22)$ and leads to an accumulation of myeloid-derived suppressor cells in the peripheral blood and bone marrow associated with immunosuppression (23). Moreover, chronic stress in mice activates the IL-10/STAT3 axis, thereby suppressing the production of pro-inflammatory cytokine IL-12 and inducing a shift of 
Th1/Th2 cytokines toward a Th2 predominance (24). Interestingly, a decreased Th1 immune response has recently been shown to lead to increased susceptibility of mice to infection with the enteropathogenic Escherichia coli (25). Based on these findings, we hypothesized that psychological factors may increase the risk to develop PI-IBS by modulation of the immune response towards the gastrointestinal infection.

In December 2010, two small villages in Belgium (Hemiksem and Schelle) suffered from a large community-wide multi-pathogen outbreak of gastroenteritis due to contamination of the tap water with water from a small creek (26). At least 18000 local residents were exposed to this contaminated water supply (26). In the present study, we aimed 1. to explore the potential relationship between psychological factors, the immune response in the acute infectious phase and the risk to develop infectious gastroenteritis and/or PI-IBS; and 2. to study in depth the potential role of persistent immune activation in those patients who developed PI-IBS. To this end, data were prospectively collected using questionnaires evaluating demographics and psychological data, exposure to tap water, bowel habits before and during the outbreak. Additionally, clinical symptoms and immune activation were assessed by validated questionnaires and quantification of cytokine expression in (stimulated) peripheral blood mononuclear cells, in plasma and in rectal biopsies respectively in the acute phase of the infection and after 1 year.

\section{MATERIAL AND METHODS:}

An epidemic outbreak of IGE occurred in Hemiksem and Schelle (Belgium) between December 6 and 9 in 2010 by contamination of the municipal tap water with local creek water as reported by the Local Health Authority (26). Although not officially established, the contamination was most likely caused by a misconnection of hoses by fireman during fire extinction. From December 6 onwards, the 18620 inhabitants of both villages were exposed to contaminated water before being alarmed at December 9 by the Local Health Authority. From December 9 onwards, the population was advised to stop drinking tap water. At December 10, a task force was set up in our unit to study the epidemiology and long-term outcome of the outbreak. The study protocol was approved by the Ethics Committee of the University Hospitals Leuven and all patients gave informed consent.

Study design:

In the acute phase (within 2 weeks), 603 subjects who consulted their general physician because of IGE were approached by phone. This subset of patients underwent a standardized interview, filled out questionnaires (details below) and was approached to provide blood samples for PBMC isolation, faecal samples and rectal biopsies. Based on questionnaires, data were collected on demographics 
(age, gender), exposure to tap water, bowel habits before and during the outbreak (ROME-III and GSRS questionnaires) and IGE characteristics, including onset of symptoms, duration of diarrhea, severity of abdominal discomfort and use of antibiotics. Moreover, to assess their psychological profile, subjects were asked to fill out the Hospital Anxiety and Depression Scale (HADS) based on how the subject felt 2 weeks before the outbreak and the Patient Health Questionnaire (PHQ-15) based on how the subject felt 4 weeks before the infection. Demographic data on the different recruitment strategies are reported in supplemental Table 3. To avoid the possibility that gastrointestinal symptoms at the time of infection influenced recall somatization scores, we modified the PHQ-15 by excluding three gastrointestinal items to create the PHQ-12 Somatic Symptom (PHQ-12 SS) scale (27). In addition, all households ( $n=7711)$ of the 2 affected villages were contacted by mailing within 3 months after the outbreak. Subjects were invited to participate in our study and to complete questionnaires as described above. Subjects were excluded when younger than 18 years, if they had pre-existing IBS, if symptoms of IGE started outside the 2 week period following the first day of drinking water contamination and if the Rome III questionnaire was incompletely answered.

One year after the outbreak, all subjects who participated previously were re-contacted to assess the development of PI-IBS and were asked to complete the following questionnaires: ROME-III, GSRS, HADS and PHQ-12. In addition, patients were asked to provide blood samples for PBMC isolation, faecal samples and rectal biopsies.

Outcome measure / definitions:

IGE was defined as a period of self-reported acute gastrointestinal discomfort within 2 weeks after tap water contamination. The severity of diarrhea was classified as follows (28): Classic IGE was defined as passage of three or more unformed stools per $24 \mathrm{~h}$ with at least one accompanying symptom (nausea, vomiting, abdominal cramps or pain, fever, blood in stools). Moderate IGE was defined as passage of one or two unformed stools with at least one additional symptom. Mild IGE was defined as passage of one or two unformed stools without additional symptoms. Controls (noIGE) were subjects who were exposed but did not report symptoms of IGE. The severity of abdominal cramping and abdominal pain was scored as none, mild, moderate or severe. IBS and the different IBS subtypes were diagnosed according to the ROME III criteria (http://www.romecriteria.org/ assets/pdf/19_RomellI_apA_885-898.pdf). Subjects who reported IBS symptoms or had been diagnosed with IBS prior to December 6th 2010 were excluded from the study. The incidence of dyspepsia-like symptoms was based on 5 GSRS questions, namely epigastric pain and discomfort, sucking sensation in the epigastrium, nausea, bloating and regurgitation. A subject was considered as dyspeptic when the summated score on these questions is larger than 4. Finally, a total HADS anxiety 
or depression score from 0 to 7 was considered normal, 8 to 10 as borderline while scores above 10 were considered to be abnormal $(29 ; 30)$.

Statistical analysis, gene expression studies, PBMC immune-phenotyping and cytokine analysis in plasma and in supernatants of stimulated PBMCs are described in supplementary Material and Methods.

RESULTS:

Characterization of patient cohort:

A. Early phase

Patient cohort

Between December $6^{\text {th }}$ and $9^{\text {th }} 2010,18620$ residents in 7711 households in Hemiksem and Schelle were exposed to contaminated tap water and asked to participate in our study. 1379 subjects completed the questionnaires of which 411 individuals were excluded (Flowchart Fig. 1).

Of the 968 eligible subjects, 271 (28\%) reported IGE: 62\% presented with classic IGE, 19\% with moderate IGE and $19 \%$ with abdominal symptoms but without diarrhea (IGE w/o diarrhea). The number of IGE cases followed a steep incline from December 6 to $8^{\text {th }}$ and peaked on December $8^{\text {th }}$ (Fig. 2). Other main IGE symptoms included nausea (reported by $54 \%$ ), vomiting (61\%), abdominal cramping (55\%) and abdominal pain (69\%). The mean duration of illness was $7.7 \pm 8.2$ days, the mean duration of diarrhea was $5.2 \pm 8.4$ days. Nine out of 270 individuals ( $3 \%, n=1$ missing data on rectal bleeding) reported rectal blood loss.

Fifty six fecal samples were collected from subjects clinically diagnosed with IGE of which 26 specimens tested positive for an infectious pathogenic agent. Norovirus NoV GI.4, GII.4, GII.14, GII.7 was most frequently detected (15/56), followed by Giardia lamblia (5/56), Campylobacter ssp. $(4 / 56)$, adenovirus (1/56) and rotavirus A (1/56). Two patients were positive for more than 1 infectious agent: 1 with NoV GI.4 and GII.4, and 1 with Campylobacter jejuni and NoV GI.4 and GII.7.

Risk factors underlying the susceptibility to develop IGE

Participants were asked to provide information on their consumption and use of the drinking water. $39 \%$ of inhabitants drank tap water with an average of 3.7 glasses per day while almost all subjects used tap water to brush their teeth (98\%) or to wash vegetables and fruit (97\%).

As previous studies indicated psychological factors as risk factor to develop PI-IBS, subjects were asked to complete the HADS and PHQ-12 questionnaires to assess anxiety, depression and somatization 2 and 4 weeks before the outbreak respectively. As shown in Fig. 3A, the proportion of subjects with abnormal anxiety scores $(>10)$ was increased in the IGE group $(11 \%)$ compared to the exposed group (4\%, p X2=0.0001). Also PHQ-12 somatization scores were increased in the IGE group 
(median=3 IQR[2-7]) compared to the exposed group (median=2.5 IQR[1-5], Mann Whitney-U test $p=0.0001$ ). The proportion of subjects with abnormal depression scores was not different between the IGE (4\%) and the exposed group (5\%) (Fig. 3A). Of note, the prevalence of pre-existing dyspepsialike symptoms before the IGE outbreak was higher in the IGE group compared to the exposed group ( $35 \%$ vs $19 \%$ respectively; $p \times 2<0.0001$ ).

As shown in Table 1, univariate analyses revealed that the development of IGE was associated with gender, age, pre-existing dyspepsia-like symptoms and drinking contaminated tap water. Of interest, anxiety and PHQ12 somatization, but not depression scores, were significantly associated with the development of IGE (Table 1, Fig. 3A). Inclusion of the significant parameters in a multivariate binary logistic regression model identified age, pre-existing dyspepsia-like symptoms, drinking tap water and anxiety as independent risk factors to develop IGE (Table 1).

Of interest, analysis of the risk factors to develop IGE based on only individuals with classic $\underline{\text { IGE ( }} \mathrm{n=168)}$ compared to the IGE- group ( $\mathrm{n}=697$ ) revealed very similar results (data not shown).

Table 1. Risk factors to develop IGE

\begin{tabular}{|c|c|c|c|c|c|c|c|}
\hline & Total & IGE+ & IGE- & $\begin{array}{c}\text { Univariate } \\
\text { Pearson } \chi^{2} \\
\mathbf{p}\end{array}$ & OR [95\% Cl] & $\begin{array}{l}\text { Multiva } \\
\text { riate } \\
\text { logistic } \\
\text { regressi } \\
\text { on } p\end{array}$ & OR [95\% Cl] \\
\hline Acute phase & 968 & 271 & 697 & $\bar{z}$ & & & \\
\hline Female, $\mathrm{N}(\%)$ & 496 (51\%) & $156(59 \%)$ & $340(49 \%)$ & 0.015 & & NS & \\
\hline Age, mean(SD) & $52(16)$ & 49 (16) & $53(16)$ & $<0.0001 \#$ & $0.98[0.98-0.99]$ & 0.001 & $0.98[0.97-0.99]$ \\
\hline $\begin{array}{l}\text { Pre-existing dyspepsia-like } \\
\text { symptoms, N (\%) }\end{array}$ & $213(22 \%)$ & $93(35 \%)$ & $120(19 \%)$ & $<0.0001$ & & 0.026 & $1.1[1.0-1.1]$ \\
\hline \multicolumn{8}{|l|}{ Exposure to tap water: } \\
\hline $\begin{array}{l}\text { Not reported, } \mathrm{N}(\%) \\
\text { Drinking tap water, } \mathrm{N}(\%)\end{array}$ & & $\begin{array}{l}87(32 \%) \\
104(57 \%)\end{array}$ & $\begin{array}{c}2(0.3 \%) \\
242(35 \%)\end{array}$ & $<0.0001$ & & $<0.0001$ & $2.6[1.8-3.7]$ \\
\hline \multicolumn{8}{|l|}{ Psychological factors: } \\
\hline $\begin{array}{l}\text { anxiety HADS score (mean, } \\
\qquad 95 \% \mathrm{Cl}]\end{array}$ & & $\begin{array}{c}5.29[4.78- \\
5.79]\end{array}$ & $\begin{array}{c}3.99[3.73- \\
4.25]\end{array}$ & $<0.0001 \#$ & $1.1[1.1-1.2]$ & 0.010 & $1.1[1.0-1.1]$ \\
\hline $\begin{array}{l}\text { depression HADS score } \\
\text { (mean, } 95 \% \mathrm{Cl}]\end{array}$ & & $\begin{array}{c}3.37[2.95- \\
3.80]\end{array}$ & $\begin{array}{c}3.19[2.93- \\
3.45]\end{array}$ & 0.62 & $1.0[0.97-1.05]$ & & \\
\hline $\begin{array}{l}\text { somatization score (PHQ-12) } \\
\text { (mean, } 95 \% \mathrm{Cl}]\end{array}$ & & $\begin{array}{c}4.50[4.05- \\
4.95]\end{array}$ & $\begin{array}{c}3.37[3.13- \\
3.62]\end{array}$ & $<0.0001 \#$ & $1.1[1.06-1.14]$ & NS & \\
\hline
\end{tabular}


Immune response in the infectious period and relationship with psychological factors

To evaluate the relationship between psychological factors and the immune response to the infection, PBMCs were immunologically phenotyped in great detail, plasma cytokines were measured and the expression of inflammatory genes was studied in rectal biopsies. Plasma cytokine analysis of

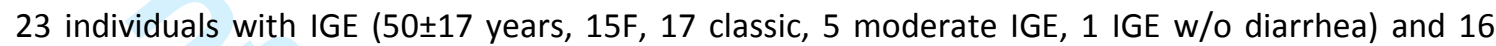
healthy controls ( $46 \pm 15$ years, 9F) revealed no differences in cytokine levels for IFNY, IL4, IL13, IL6, IL8, IL10, IL13 or IL17 (sFig.1). In contrast, inflammatory gene mRNA expression for IL10, TNF $\alpha$, IL6, MCP-1 and IFN $\gamma$ was significantly upregulated in rectal biopsies of 9 subjects with IGE (mean age $44 \pm 16$ years, 5F) compared to 9 healthy controls (mean age $54 \pm 17$ years, 5F) (Fig. 3C). mRNA expression levels for IL4 and IL5 were below detection limit.

Next, we evaluated the potential relationship between psychological factors and the immune response to the infectious pathogens in the total IGE group. The number of CD4+CD8-IL2+ T cells, a cell type critical in maintaining regulatory $T$ cells (Treg)(31), was inversely correlated with anxiety, depression and somatization (Fig. 3B). The observed significances for CD4+CD8-IL2+ T cells did not survive FRD correction for multiple testing. There was no correlation between psychological scores and Tregs, Th1, Th2 or Th17 cells (data not shown). 
B. One year follow-up

Incidence of IBS and type of gastrointestinal symptoms:

567 of the 968 (59\%) participants returned the questionnaires 1 year after the outbreak, including $180 / 271$ (66\%) of the IGE group and 387/697 (56\%) of the exposed subjects who did not develop IGE (Fig. 1). 29 subjects (8 IGE, 21 exposed) were excluded because of incomplete Rome questionnaires (Fig. 1). 19.8\% (34/172) of IGE subjects developed IBS (IGE+IBS+, OR: 3.0[1.8-4.9]) compared to $6.6 \%$ (24/366) of the exposed group that did not develop IGE (IGE-IBS+, pX2<0.0001). Of the PI-IBS patients, $24 \%$ had IBS-C, 35\% IBS-D, 6\% IBS-M and 35\% IBS-U. The main gastrointestinal symptoms, assessed using the GSRS questionnaire, are shown in Supplemental Figure 2. Notably, IGE patients also developed more dyspeptic symptoms since the outbreak; $25 \%$ of IGE patients developed an abnormal dyspepsia-like symptom score compared to $14 \%$ in the exposed no-IGE group (px2<0.003, OR: 2.0 [1.3-3.3]). Demographic data on subjects who responded versus who did not at 1 year follow-up are reported in supplemental Table 4.

\section{Psychological factors and immune profile 1 year after the outbreak}

The proportion of subjects with an abnormal anxiety score was higher in the IGE subjects whether they did (IGE+IBS+12\%) or did not develop IBS (IGE+IBS-, 9\%) compared to the exposed group and in subjects that developed IBS but had no IGE (IGE-IBS+, 25\%) compared to the group that did not develop IGE or IBS (exposed IBS-, 3\%, p X2<0.0001, Fig. 4A). PHQ-12 somatization scores were also increased in the PI-IBS- (median: 6 IQR[5-7]), PI-IBS+ (median: 5 IQR[5-6]) and exposed IBS+ (median=5.5 IQR[5-6]) groups compared to the exposed IBS- group (median=3 IQR[1-5], p Kruskal Wallis with Dunns' correction for multiple comparisons) (Fig. 4A). In contrast, the proportion of subjects with abnormal depression scores was similar amongst groups (Fig. 4A).

To evaluate the role of persistent immune activation in the development of PI-IBS, PBMC phenotypes and plasma cytokine levels were assessed in 108 individuals (for details, see Supplemental Table 2), including 17 PI-IBS+. CD4-CD19+ B cells were increased in IGE+IBS+ compared to exposed IBS- individuals (Fig. $4 \mathrm{~B}$ ). However, there were no differences in B cell subpopulations (sFig. 2), immuno-phenotypes of T cell populations, DCs or NK cells when comparing IGE+IBS+ with IGE+IBS- and IGE-IBS- subjects. When assessing cytokine levels in supernatants of stimulated PBMCs ( $n=77$, supplemental Table 2), IL10 and IL-8 levels were increased in PI-IBS compared to the exposed group while IL13, IL1b and TNF $\alpha$ levels were similar in all groups (Fig. 4C). Plasma cytokine levels (IFNg, IL17, IL10, IL4, IL6, IL8 and IL13 were not different between IGE+IBS+ and IGE+IBS- or exposed groups (sFig. 4). None of the results could withstand Bonferroni multiple testing correction. 
Finally, inflammatory cytokines mRNA was assessed in rectal mucosal biopsies of 32 subjects, including 9 IGE+IBS-, 5 IGE+IBS+, 15 IGE-IBS- and 3 IGE-IBS+ (sTable 3). There was no difference in mRNA expression level of IL10, IL6, IL8, TNFa nor hMCP-1 between IGE+IBS+, IGE+IBS- and exposed groups (sFig. 5). IFN $\gamma$ and IL5 mRNA expression levels were below detection limits ( $\mathrm{Ct}=35$ ).

\section{Risk factors to develop PI-IBS}

As shown in Table 2, univariate analysis revealed that the development of PI-IBS after IGE is associated with anxiety, somatization and GSRS dyspepsia-like symptom scores, all prior to outbreak and at follow-up, together with age and IGE severity (number of diarrhea days, abdominal cramping).

Table 2. Risk factors to develop PI-IBS after IGE

\begin{tabular}{|c|c|c|c|c|c|}
\hline & Total & $\begin{array}{c}\text { Self-reported } \\
\text { IGE }\end{array}$ & $\begin{array}{l}\text { Exposed, no } \\
\text { IGE }\end{array}$ & $\begin{array}{l}\text { Pearson } \chi^{2} p \text { IGE } \\
\text { vs no IGE }\end{array}$ & \\
\hline 1 year follow-up & 538 & 172 & 366 & & \\
\hline Female, $\mathrm{N}(\%)$ & $258(48 \%)$ & $92(53 \%)$ & $166(45 \%)$ & 0.08 & \\
\hline Age, mean (SD) & $52(15)$ & $50(15)$ & $54(15)$ & $0.016 \#$ & \\
\hline \multirow[t]{2}{*}{ IBS, N (\%) } & $58(11 \%)$ & $34(20 \%)$ & $24(7 \%)$ & $<0.0001$ & \\
\hline & PI-IBS & IGE & $\begin{array}{c}\text { Univariate } \\
\text { binary logistic } \\
\text { regression test } \\
\text { p }\end{array}$ & $\begin{array}{l}\text { Multivariate } \\
\text { logistic } \\
\text { regression p }\end{array}$ & OR [95\% Cl] \\
\hline \multicolumn{6}{|l|}{ Risk factors PI-IBS acute phase } \\
\hline Age, mean(SD) & $42.4(13.2)$ & $52.0(15.3)$ & 0.002 & 0.010 & $0.95[0.91-0.99]$ \\
\hline $\begin{array}{l}\text { Number of diarrhea days, mean } \\
\qquad \text { (SD) }\end{array}$ & $7.8(7.4)$ & $4.7(5.4)$ & 0.03 & NS & \\
\hline Abdominal cramping & & & 0.017 & NS & \\
\hline HADS anxiety & & & 0.014 & NS & \\
\hline PHQ12 somatization & & & 0.0001 & 0.008 & $1.16[1.03-1.31]$ \\
\hline $\begin{array}{l}\text { Pre-existing dyspepsia-like } \\
\text { symptoms }\end{array}$ & & & 0.013 & NS & \\
\hline \multicolumn{6}{|l|}{$\begin{array}{l}\text { Risk factors PI-IBS at } 1 \mathrm{yr} \\
\text { follow-up }\end{array}$} \\
\hline HADS anxiety & & & 0.044 & NS & \\
\hline PHQ12 somatization & & & $<0.0001$ & NS & \\
\hline Dyspepsia-like symptoms & & & $<0.0001$ & 0.0001 & $1.17[1.05-1.30]$ \\
\hline
\end{tabular}

\# $\mathrm{p}$ binary logistic regression test, NS: not significant in multivariate analysis 
In contrast, the development of PI-IBS was not associated with gender, other IGE characteristics, drinking tap water or number of glasses water consumed per day, use of medication (antibiotics, anti-diarrhea, ORS), allergy (hay fever, eczema), asthma, atopic constitution, alcohol, smoking, drugs, or familial history of IBD/IBS, prior major depression or traumatic life time experience, depression at time of outbreak or at follow-up. The multivariate logistic regression model at the time of infection revealed age and somatization as risk factor to develop PI-IBS. Inclusion of the significant parameters at 1 year follow-up identified the presence of dyspepsia-like symptoms as independent factor associated with PI-IBS (Table 2).

To study the relative contribution of psychological factors and IGE as risk factors to develop IBS, we performed an extra analysis on all subgroups (IGE-IBS-, IGE-IBS+, IGE+IBS- and IGE+IBS+). In line with our findings above, anxiety before the outbreak predicts IGE development $(p=0.001$, path $b$ in Fig. 5A) and IBS at follow-up ( $p<0.0001$, Fig. 5B) directly. When IGE status was added to the model in Fig. $5 B$, both anxiety $(p<0.0001$, path a' in Fig. $5 A)$ and IGE $(p=0.0018$, path $c$ in Fig. $5 A)$ were identified as predictors of IBS at follow-up, without the direct effect of anxiety being changed compared to the model in Fig. 5B. Therefore, the effect of anxiety on IBS at follow-up is not only direct but also to a limited though significant extent indirect through increasing vulnerability to IGE development. Similarly, somatization scores before the outbreak predicted IGE $(p=0.0088$, path $b$ in Fig. $5 C$ ) and IBS ( $p<0.0001$, path a in Fig. 5D). In combination, both somatization ( $p<0.0001$, path $a^{\prime}$ in Fig. 5C) and IGE (path c in Fig. 5C) are significant predictors of IBS at follow-up, without the effect of somatization being significantly changed compared to the model in Fig. 5D. This indicates that the effect of somatization on IBS is not only direct, but also to a certain extent indirect through increased risk of IGE during the outbreak.

Finally, depression before the outbreak predicts IBS ( $p=0.0038$, path a in Fig. $5 F$ ) but not the development of IGE ( $p=0.15$, path $b$ in Fig. $5 E$ ). When IGE status was added to the model, both depression ( $p=0.0077$, path $a^{\prime}$ in Fig. $5 E$ ) and IGE ( $p=0.0002$, path $c$ in Fig. $5 E$ ) were predictors of IBS at follow-up, without the effect of depression being changed compared to $F$, suggesting that the effects of depression and IGE on the development of IBS are independent of each other.

To evaluate if the acute immune response during the episode of IGE predicts the development of PI-IBS, the phenotype of the PBMCS of PI-IBS patients in the acute phase was compared with those who did not develop PI-IBS. Although the $n$-values are small, increased numbers of Th2 cells were observed at the time of IGE in individuals who went on to develop PI-IBS compared to those who did not ( $p=0.02$, Mann Whitney $U$ t-test, Fig. 6$)$. There is also a trend towards decreased numbers of Th1 cells and CD4+CD8-IL2+ cells $(p=0.07)$ during IGE in subjects who develop 
PI-IBS 1 year later compared to those who do not (Fig. 6). Plasma cytokine levels (IFNg, IL17, IL10, IL4, IL6, IL8 and IL13) were not different (data not shown).

\section{DISCUSSION}

A large cohort exposed to contaminated drinking water was prospectively studied to assess the risk to develop PI-IBS, focusing on the role of psychological factors and the immune response both in the acute phase and 1 year after the outbreak. Our study indicates that IGE and psychological factors (anxiety and somatization but not depression) are associated with an increased risk to develop PI-IBS. Of interest though, we showed that anxiety and somatization are also risk factors to develop IGE, thereby indirectly adding to the increased risk to develop IBS. Thorough analysis of the immune response in the acute IGE phase revealed that anxiety was associated with a reduction in IL2 expressing $C D 4+C D 8-T$ cells, suggesting a link between psychological factors, immune response and the observed increased risk to develop IGE. Moreover, we found that patients who developed PI-IBS had a lower number of CD4+CD8- IL2+ T cells and Th1 cells but an increased number of Th2 cells at the time of infection. These data suggest that a predominant Th2 response against the infectious insult may predispose to progression to PI-IBS. The resolution of the systemic Th2 bias by one year follow-up is consistent with a shift in pathophysiology to the mucosa, and may explain why cohort studies have found conflicting results when determining whether IBS patients have a systemic Th2 bias $(14 ; 32 ; 33)$. Based on these findings, we propose that psychological factors, in particular anxiety and somatization, increase the risk to develop PI-IBS. This effect is to a certain extent explained by increasing the susceptibility to develop IGE, possibly by skewing the immune response towards a predominant Th2 response at the time of infection.

An episode of infectious gastroenteritis is a well-defined risk factor to develop IBS (34). Here, we report on a large cohort of more than 18000 residents exposed to drinking water contaminated by creek water containing Norovirus, Campylobacter spp and Giardia lamblia. Of the 968 residents who participated, $28 \%$ developed symptoms of IGE. Similar to previous studies (8), IGE was associated with an increased risk (odds ratio of 3 ) to develop PI-IBS, i.e. $20 \%$ of IGE patients was identified as IBS compared to $7 \%$ in the exposed control group. This proportion is higher compared to the $8 \%$ reported following a food-borne outbreak with Salmonella (3), but lower than the $36 \%$ detected in the mixed waterborne outbreak of Walkerton (9). The proportion of IBS-C ( $35 \%$ in the PIIBS group compared to less than $10 \%$ in literature) tends to be higher compared to previous studies $(35 ; 36)$. The explanation for this difference remains speculative but the type and localization of infection may be factors that determine the clinical phenotype. In addition to severity of IGE and age, and consistent with previous studies (2;12), psychological factors, in particular anxiety and 
somatization, were identified as risk factors to develop IBS (37) and PI-IBS (2;12). A novel and intriguing finding, however, is that anxiety and somatization are also associated with an increased susceptibility to develop IGE. This observation suggests that the increased risk of subjects with increased anxiety and somatization to develop PI-IBS is partly explained by the observation that they are more susceptible for a gastrointestinal infection. To what extent this results from stress-induced impaired barrier function (38) or impaired immune function with hampered clearance of the infectious agent (25) is hard to investigate. Of note, age is protective against IGE and this may possibly be directly related to age or may be due to previous exposure. Moreover, at the time of infection, anxiety and depression were inversely correlated with the percentage of CD4+CD8- IL-2producing T cells in peripheral blood. In line, subjects with IGE who developed PI-IBS also had low numbers of $C D 4+C D 8-I L-2+T$ cells at the time of infection. Moreover, these individuals revealed a more Th2 skewed immune response during the infectious challenge, as evidence by increased Th2 cells and reduced Th1 cells in the blood samples collected during the period of IGE. As IL-2 is an important Th1 cytokine and CD4+CD8-IL2+ T cells are involved in regulatory $T$ cell biology and immune homeostasis (31), the reduction in CD4+IL-2+ T cells related to anxiety and depression may theoretically contribute to the increased susceptibility for infection and perhaps set the stage to develop PI-IBS. It should be emphasized though that the number of blood samples studied is low, and that this conclusion thus remains very speculative. Nevertheless, animal studies support the notion that psychological factors can drive the Th1/Th2 balance towards Th2 predominance (24), and thereby increase susceptibility to infection with enteropathogens (25).

Persistent mucosal microscopic inflammation with increased mast cells, lamina propria T cells and enterochromaffin cells has been demonstrated in intestinal biopsies collected from PI-IBS patients (2;13;39). In addition, Gwee et al. (13) showed increased IL-1beta expression in PI-IBS rectal biopsies taken 3 months after the infection. However, we failed to detect any changes in expression of pro-inflammatory cytokines in rectal biopsies of collected 1 year after infection. Similar findings were reported by Mearin et al (14) although it has to be emphasized that patients were studied three years after gastroenteritis. Our results may be explained by the fact that the infectious pathogens involved (noroviruses, Giardia lamblia and Campylobacter spp) do not infect the distal gut and thus may not induce long-term immunological changes in the rectum. However, we did find upregulation of pro-inflammatory cytokines in the acute infectious phase arguing against this possibility. Moreover, we recently found that submucosal neurons of rectal biopsies of IGE+IBS patients from this cohort were more sensitive to the TRPV1 agonist capsaicin compared to biopsies of the IGE+IBSgroup (40). In line, Mearin et al. showed hypersensitivity to rectal distention in PI-IBS patients compared to infected patients who did not develop IBS, even though no differences were found in 
rectal biopsies. Taken together, these data indicate that intestinal sensory function can indeed be affected by an episode of IGE, even in the absence of local persistent inflammation.

To further evaluate the role of immune activation in PI-IBS, we also thoroughly phenotyped PBMCs and quantified plasma cytokine levels. Of interest, we found increased CD4-CD19+ B cell numbers in PI-IBS+ compared to PI-IBS- patients. Our finding that PI-IBS patients have a Th2 predominant immune response at the time of infection, is compatible with the assumption that a humoral immune response could be involved in PI-IBS. This hypothesis is indirectly supported by 2 recent studies in IBS patients showing increased activation of mucosal B lymphocytes and plasma cells in jejunal biopsies (41) and increased activation level of blood B cells (42). Similar to previous studies in IBS patients $(43 ; 44)$ we also found an increase in serum IL-8 levels, however, no other signs of aberrant immune activation in PI-IBS were observed.

The major strength of our study is that we prospectively studied a community sample of exposed and infected subjects and assessed psychological and immunological factors in great detail both in the acute infectious phase and 1 year later. This allowed us to identify psychological factors not only as a risk factor to develop PI-IBS but also to unravel its impact on the susceptibility to develop IGE. Moreover, this is the first study reporting on the immune response during the period of IGE, albeit in a small group of subjects, and to relate this to the risk to develop PI-IBS 1 year later. The major weakness of the study is the relatively low sample size of rectal biopsies and blood samples, in particular in the acute infectious phase. As a result, some of our data do not withstand correction for multiple testing, and should be interpreted cautiously. Also, the low recruitment number may introduce some bias and therefore extrapolation of our data to a wider population should be done with caution. This low sample number is inherent to the fact that it is a prospective study and it is logistically difficult to recruit individuals during the acute period of the outbreak. Moreover, our study is limited by the fact that questionnaires on anxiety, depression and somatization before the infection were assessed in the acute infectious phase and we cannot exclude a recall bias due to gastrointestinal symptoms experienced due to the infection. In an attempt to circumvent this problem, we excluded questionnaires on gastrointestinal symptoms from the PHQ15 questionnaire.

Altogether, our study shows that the increased risk of patients with psychological comorbidity to develop PI-IBS is partly due to an increased susceptibility to develop IGE. Moreover, we provide evidence to speculate that subjects who develop a predominant Th2 response against the infectious agent are prone to develop a humoral immune response and subsequently PI-IBS. 


\section{CONFLICTS OF INTEREST}

The authors declare no conflict of interest

\section{AUTHOR CONTRIBUTIONS:}

All authors read and approved the final version of the manuscript.

Mira M Wouters: $\quad$ data acquisition, analysis and interpretation of data, writing and

\begin{tabular}{|c|c|}
\hline Anh Nguyen: & $\begin{array}{l}\text { critical revision of the manuscript for important intellectual content } \\
\text { data acquisition, analysis and interpretation of data }\end{array}$ \\
\hline Sander JM van Wanrooij: & $\begin{array}{l}\text { study concept and design, acquisition of data, analysis and } \\
\text { interpretation of data }\end{array}$ \\
\hline James Dooley: & data acquisition \\
\hline Javier Aguilera-Lizarra & data acquisition \\
\hline Winde Vanbrabant: & recruitment of study subjects, data acquisition \\
\hline Josselyn E Garcia-Perez: & data acquisition \\
\hline Lukas Van Oudenhove: & $\begin{array}{l}\text { statistical analysis, interpretation of data, critical revision of the } \\
\text { manuscript for important intellectual content }\end{array}$ \\
\hline Marc Van Ranst: & data acquisition \\
\hline Jan Verhaegen: & data acquisition \\
\hline Adrian Liston: & $\begin{array}{l}\text { study supervision, obtaining funding, critical revision of the } \\
\text { manuscript for important intellectual content }\end{array}$ \\
\hline uy Boeckxstaens: & $\begin{array}{l}\text { study supervision, obtaining funding, critical revision of the } \\
\text { manuscript for important intellectual content }\end{array}$ \\
\hline
\end{tabular}

FUNDING: GEB was funded by a governmental (Odysseus programme, G.0905.07) of the Research Foundation - Flanders (FWO) and university grant (Global Opportunities for Associations GOA 14.011). MMW is supported by a FWO postdoctoral fellowship. This work was funded by FWO research grant G.0699.10N to GB and MMW. AL received the ERC Start grant IMMUNO. TLAN is funded by VIB International PhD program.

TRIAL REGISTRATION: ClinicalTrials.gov ID: NCT01497847 entitled "Post-Infectious Irritable Bowel Syndrome (PI-IBS)" 


\section{FIGURE LEGENDS}

Figure 1. Flow chart of outbreak cohort

Figure 2. Incidence of gastroenteritis

Individuals who developed IGE between December 6 and 19 were included in the study.

Figure 3. Prevalence of psychological conditions prior to infectious gastroenteritis and correlation with IL2 expressing T cells in acute infectious phase

Panel A: Psychological status 2 weeks before the outbreak was assessed by HADS-anxiety and depression questionnaires and the PHQ12 somatization questionnaire. Panel B: HADS-anxiety, depression and somatization were inversely correlated with IL2 expressing CD4+ cells. Panel C: Relative mRNA expression levels of inflammatory genes in rectal biopsies were increased in subjects with IGE compared to non-infected, healthy volunteers (HV; relative expression to HPRT1 housekeeping gene). Infected subjects suffered significantly more from increased anxiety and somatization compared to exposed subjects who would not develop symptoms of gastroenteritis (no IGE).

Figure 4. Psychological factors, PBMC immunophenotypes and cytokine analysis of stimulated PBMCs at 1 year follow-up

Panel A: HADS-anxiety and depression and PHQ12 somatization were assessed 1 year after the outbreak. Both IBS groups (IGE+IBS+ and IGE-IBS+) showed increased levels of anxiety and somatization compared to healthy individuals. Panel $B$ : T cell phenotypes and $B$ cell numbers were assessed and compared between subjects who developed IBS compared to subjects who did not. Only B cell numbers were significantly increased in PI-IBS patients compared to the exposed IBSsubjects. Panel C: Cytokine release of stimulated mononuclear leukocytes (PBMC) was assessed as a measure of their activation in vivo.

PI NS: post-infectious no symptoms, ${ }^{*} p<0.05 ;{ }^{* *} p<0.001 ;{ }^{* * *} p<0.0001$

Figure 5. The effect of psychological factors on the susceptibility to develop infectious gastroenteritis and IBS symptoms

The effect of anxiety, somatization and depression was assessed on the development of infectious gastroenteritis and IBS symptoms. Fig. A, C and $E$ show the indirect effect of psychological factors on the development of IBS symptoms via increasing the vulnerability to gastroenteritis. Fig. B, C and $F$ show the direct effect of psychological factors on the development of IBS symptoms.

Figure 6. T helper cell analysis in the acute infectious phase comparing individuals who will develop PI-IBS compared to who will become symptom free

Infected individuals who will develop PI-IBS (PI-IBS+) show a tendency for Th2 skewing towards the infectious pathogens as shown by increased Th2 numbers and a tendency toward decreased Th1 and CD4+CD8- IL expressing cells compared to infected individuals who will become symptom free (PIIBS-). ${ }^{*} \mathrm{p}<0.05$, Mann Whitney U t-test 
(1) Spiller RC, Jenkins D, Thornley JP et al. Increased rectal mucosal enteroendocrine cells, T lymphocytes, and increased gut permeability following acute Campylobacter enteritis and in post-dysenteric irritable bowel syndrome. Gut 2000;47(6):804-11.

(2) Dunlop SP, Jenkins D, Neal KR et al. Relative importance of enterochromaffin cell hyperplasia, anxiety, and depression in postinfectious IBS. Gastroenterology 2003;125(6):1651-9.

(3) Mearin F, Perez-Oliveras M, Perello A et al. Dyspepsia and irritable bowel syndrome after a Salmonella gastroenteritis outbreak: one-year follow-up cohort study. Gastroenterology 2005;129(1):98-104.

(4) McKendrick MW, Read NW. Irritable bowel syndrome--post salmonella infection. J Infect 1994;29(1):1-3.

(5) Porter CK, Faix DJ, Shiau D et al. Postinfectious gastrointestinal disorders following norovirus outbreaks. Clin Infect Dis 2012;55(7):915-22.

(6) Zanini B, Ricci C, Bandera F et al. Incidence of post-infectious irritable bowel syndrome and functional intestinal disorders following a water-borne viral gastroenteritis outbreak. Am J Gastroenterol 2012;107(6):891-9.

(7) Wensaas KA, Langeland N, Hanevik K et al. Irritable bowel syndrome and chronic fatigue 3 years after acute giardiasis: historic cohort study. Gut 2012;61(2):214-9.

(8) Thabane M, Kottachchi DT, Marshall JK. Systematic review and meta-analysis: The incidence and prognosis of post-infectious irritable bowel syndrome. Aliment Pharmacol Ther 2007;26(4):535-44.

(9) Marshall JK, Thabane M, Garg AX et al. Incidence and epidemiology of irritable bowel syndrome after a large waterborne outbreak of bacterial dysentery. Gastroenterology 2006;131(2):445-50.

(10) Marshall JK, Thabane M, Garg AX et al. Eight year prognosis of postinfectious irritable bowel syndrome following waterborne bacterial dysentery. Gut 2010;59(5):605-11.

(11) Ford AC, Talley NJ. Mucosal inflammation as a potential etiological factor in irritable bowel syndrome: a systematic review. J Gastroenterol 2011;46(4):421-31.

(12) Gwee KA, Leong YL, Graham C et al. The role of psychological and biological factors in postinfective gut dysfunction. Gut 1999;44(3):400-6.

(13) Gwee KA, Collins SM, Read NW et al. Increased rectal mucosal expression of interleukin 1 beta in recently acquired post-infectious irritable bowel syndrome. Gut 2003;52(4):523-6. 
(14) Mearin F, Perello A, Balboa A et al. Pathogenic mechanisms of postinfectious functional gastrointestinal disorders: results 3 years after gastroenteritis. Scand J Gastroenterol 2009;44(10):1173-85.

(15) Spiller RC. Role of infection in irritable bowel syndrome. J Gastroenterol 2007;42 Suppl 17:41-7.

(16) Dunlop SP, Jenkins D, Neal KR et al. Relative importance of enterochromaffin cell hyperplasia, anxiety, and depression in postinfectious IBS. Gastroenterology 2003;125(6):1651-9.

(17) Marshall JK, Thabane M, Garg AX et al. Eight year prognosis of postinfectious irritable bowel syndrome following waterborne bacterial dysentery. Gut 2010;59(5):605-11.

(18) Neal KR, Barker L, Spiller RC. Prognosis in post-infective irritable bowel syndrome: a six year follow up study. Gut 2002;51(3):410-3.

(19) Chiappelli F, Manfrini E, Franceschi C et al. Brain Corticosteroid Receptors: Studies on the Mechanism, Function, and Neurotoxicity of Corticosteroid Action. Proceedings of a conference. Arlington, Virginia, March 2-5, 1994. Ann N Y Acad Sci 1994;746:1-499.

(20) Dragos D, Tanasescu MD. The effect of stress on the defense systems. J Med Life 2010;3(1):10-8.

(21) Yang PC, Jury J, Soderholm JD et al. Chronic Psychological Stress in Rats Induces Intestinal Sensitization to Luminal Antigens. Am J Pathol 2006;168(1):104-14.

(22) Shaashua L, Sominsky L, Levi B et al. In vivo suppression of plasma IL-12 levels by acute and chronic stress paradigms: potential mediating mechanisms and sex differences. Brain Behav Immun 2012;26(6):996-1005.

(23) Jin J, Wang X, Wang Q et al. Chronic psychological stress induces the accumulation of myeloid-derived suppressor cells in mice. PLOS ONE 2013;8(9):e74497.

(24) Hu D, Wan L, Chen M et al. Essential role of IL-10/STAT3 in chronic stress-induced immune suppression. Brain Behav Immun 2014;36:118-27.

(25) Sharma R, Kapila R, Dass G et al. Improvement in Th1/Th2 immune homeostasis, antioxidative status and resistance to pathogenic $E$. coli on consumption of probiotic Lactobacillus rhamnosus fermented milk in aging mice. Age (Dordr) 2014;36(4):9686.

(26) Braeye T, DE SK, Wollants E et al. A large community outbreak of gastroenteritis associated with consumption of drinking water contaminated by river water, Belgium, 2010. Epidemiol Infect 2014;1-9.

(27) Spiller RC, Humes DJ, Campbell E et al. The Patient Health Questionnaire 12 Somatic Symptom scale as a predictor of symptom severity and consulting behaviour in patients with irritable bowel syndrome and symptomatic diverticular disease. Aliment Pharmacol Ther 2010;32(6):811-20.

(28) von SF, Tornieporth N, Waiyaki P et al. Risk and aetiology of diarrhoea at various tourist destinations. Lancet 2000;356(9224):133-4. 
(29) Zigmond AS, Snaith RP. The hospital anxiety and depression scale. Acta Psychiatr Scand 1983;67(6):361-70.

(30) Bjelland I, Dahl AA, Haug TT et al. The validity of the Hospital Anxiety and Depression Scale. An updated literature review. J Psychosom Res 2002;52(2):69-77.

(31) Amado IF, Berges J, Luther RJ et al. IL-2 coordinates IL-2-producing and regulatory T cell interplay. J Exp Med 2013;210(12):2707-20.

(32) Hughes PA, Zola H, Penttila IA et al. Immune activation in irritable bowel syndrome: can neuroimmune interactions explain symptoms? Am J Gastroenterol 2013;108(7):1066-74.

(33) Liebregts T, Adam B, Bredack C et al. Immune activation in patients with irritable bowel syndrome. Gastroenterology 2007;132(3):913-20.

(34) Grover M, Camilleri M, Smith K et al. On the fiftieth anniversary. Postinfectious irritable bowel syndrome: mechanisms related to pathogens. Neurogastroenterol Motil 2014;26(2):156-67.

(35) Neal KR, Barker L, Spiller RC. Prognosis in post-infective irritable bowel syndrome: a six year follow up study. Gut 2002;51(3):410-3.

(36) Dunlop SP, Jenkins D, Spiller RC. Distinctive clinical, psychological, and histological features of postinfective irritable bowel syndrome. Am J Gastroenterol 2003;98(7):1578-83.

(37) Nicholl BI, Halder SL, Macfarlane GJ et al. Psychosocial risk markers for new onset irritable bowel syndrome--results of a large prospective population-based study. Pain 2008;137(1):147-55.

(38) Yang PC, Jury J, Soderholm JD et al. Chronic psychological stress in rats induces intestinal sensitization to luminal antigens. Am J Pathol 2006;168(1):104-14.

(39) Lee KJ, Kim YB, Kim JH et al. The alteration of enterochromaffin cell, mast cell, and lamina propria T lymphocyte numbers in irritable bowel syndrome and its relationship with psychological factors. J Gastroenterol Hepatol 2008;23(11):1689-94.

(40) Balemans, D., Alpizar, Y. A., Nasser, Y., Valdez-Morales, E. E., Moonen, A., Cirillo, C., Vanner, S. J., Talavera, K., Vanden Berghe, P., Wouters, M. M., and Boeckxstaens, G. E. Evidence for Histamine-Mediated Sensitization of TRPV1 Signaling in Sensory Neurons in Mice and IBS Patients. Gastroenterology. 2014. Ref Type: Abstract

(41) Vicario M, Gonzalez-Castro AM, Martinez C et al. Increased humoral immunity in the jejunum of diarrhoea-predominant irritable bowel syndrome associated with clinical manifestations. Gut 2014.

(42) Ohman L, Lindmark AC, Isaksson S et al. B-cell activation in patients with irritable bowel syndrome (IBS). Neurogastroenterol Motil 2009;21(6):644-50, e27.

(43) McKernan DP, Gaszner G, Quigley EM et al. Altered peripheral toll-like receptor responses in the irritable bowel syndrome. Aliment Pharmacol Ther 2011;33(9):1045-52. 
(44) Scully P, McKernan DP, Keohane J et al. Plasma cytokine profiles in females with irritable bowel syndrome and extra-intestinal co-morbidity. Am J Gastroenterol 2010;105(10):2235-

43. 


\section{Psychological co-morbidity increases the risk for post-infectious IBS partly by enhanced susceptibility to develop infectious gastroenteritis}

Mira Wouters 1\&, Sander Van Wanrooy 1\&, Anh Nguyen 2,3\&, James Dooley 2,3, Javier AguileraLizarraga 1, Winde Van Brabant 1, Josselyn E Garcia-Perez 2,3, Lukas Van Oudenhove 1, Marc Van Ranst 4, Jan Verhaegen 5, Adrian Liston 2,3*, Guy Boeckxstaens 1*

\& MW, SVW and AN should be considered as first authors with equal contribution.

${ }^{*} \mathrm{AL}$ and $\mathrm{GB}$ share senior authorship.

1 Translational Research Center for Gastrointestinal Disorders, Dept. of Clinical and Experimental Medicine, University Hospital Leuven, KU Leuven, Leuven, Belgium

2 Department of Microbiology and Immunology, KU Leuven, Leuven, Belgium

3 Autoimmune Genetics Laboratory, VIB, Leuven, Belgium

4 Laboratory of Clinical Virology, Rega Institute for Medical Research, Catholic University of Leuven, Leuven, Belgium

5 Department of Microbiology, University Hospital Leuven, KU Leuven, Leuven, Belgium

Correspondence address:

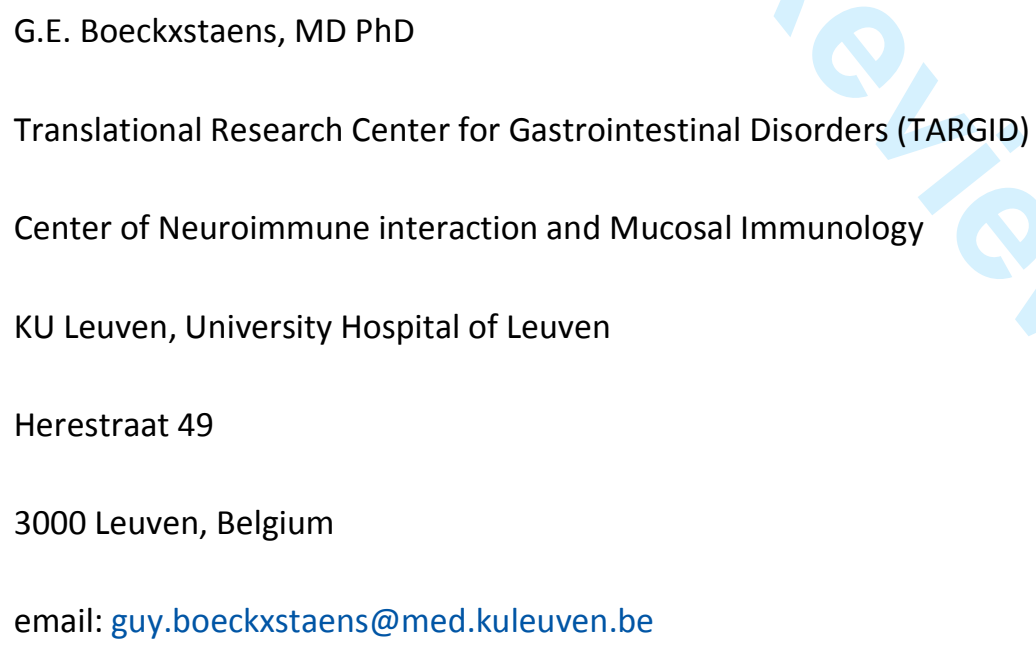

Key words: post-infectious irritable bowel syndrome, PI-IBS, Th2, anxiety 
Word count: 4708

The Corresponding Author has the right to grant on behalf of all authors and does grant on behalf of all authors, an exclusive licence (or non exclusive for government employees) on a worldwide basis to the BMJ Publishing Group Ltd and its Licensees to permit this article (if accepted) to be published in Gut editions and any other BMJPGL products to exploit all subsidiary rights, as set out in our licence 


\section{ABSTRACT:}

Objective: Psychological factors increase the risk to develop post-infectious irritable bowel syndrome (PI-IBS), but the mechanisms involved are unclear. As stress affects the immune system, we investigated the potential interaction between psychological factors, the immune response against infectious gastroenteritis (IGE) and the development of IGE and PI-IBS in a large cohort exposed to contaminated drinking water.

Design: 18620 people exposed to contaminated drinking water (Norovirus, Giardia lamblia, Campylobacter jejuni) were invited to participate in a prospective controlled cohort study. They were asked to complete questionnaires assessing demographic, psychological and clinical data during the outbreak and one year later. At both time points, in-depth immune function (peripheral blood and rectal biopsies) was studied in a subgroup of subjects.

Results: 1379 subjects completed the questionnaires during the outbreak, of which 271 developed IGE. Risk factors for IGE included younger age, pre-existing dyspepsia-like symptoms, anxiety and drinking contaminated tap water. Anxiety scores before the outbreak inversely correlated with IL2expressing CD4+Tcells ( $r=0.6, p=0.01, n=23)$. At follow-up, 34 of $172(20 \%)$ IGE subjects developed IBS compared to $24 / 366$ exposed participants ( $7 \%, p<0.0001, \chi 2$ test). A Th2 cytokine phenotype at time of infection was associated with increased risk for PI-IBS 1 year later. Except for increased B cell numbers, no evidence for systemic or rectal mucosal immune activation in PI-IBS was demonstrated at follow-up.

Conclusion: Our study shows that the increased risk of patients with psychological co-morbidity to develop PI-IBS may partly result from an increased susceptibility to develop IGE, possibly resulting from a Th2-immune bias. 


\section{SUMMARY BOX:}

What is already known about this subject:

- Up to $30 \%$ of individuals develop PI-IBS following infectious gastroenteritis

- Psychological factors increase the susceptibility to develop PI-IBS

- Psychological stress alters the Thelper 1 and T helper 2 (Th1/Th2) immune balance

What are the new findings:

- Anxiety and somatization are risk factors to develop IGE

- Psychological factors, in particular anxiety and somatization, increase the risk to develop PIIBS partly by a direct effect and partly indirect via increased susceptibility to develop IGE

- Anxiety and depression at the time of infection may alter the Th1/Th2 immune balance, predisposing to $\mathrm{PI}-\mathrm{IBS}$

- Except for increased B cell numbers, there was no evidence for persistent immune activation in blood or rectal biopsies of PI-IBS

\section{How might it impact on clinical practice in the foreseeable future?}

- Our results provide further insight in the pathogenesis of PI-IBS, underscoring the impact of psychological factors

- The absence of inflammation in PI-IBS suggests that anti-inflammatory treatment with agents such as mesalazine are unlikely to be effective 


\section{INTRODUCTION:}

Acute infectious gastroenteritis (IGE) is a well-defined risk factor to develop irritable bowel syndrome (IBS), with $8-31 \%$ of patients developing post-infectious IBS (PI-IBS) (1-10). To date, persistent mucosal microscopic inflammation is proposed to underlie abnormal neuromuscular function contributing to altered bowel habits and increased abdominal pain (11). Increased number of mononuclear cells and higher levels of IL1 $\beta$ mRNA have indeed been reported in rectal biopsies collected 3 months to 1 year after IGE (12;13). In line, 3 months after a Campylobacter infection, Dunlop et al. (2) showed a small increase in enterochromaffin cells in PI-IBS patients compared to infected subjects who did not develop IBS. However, the number of lamina propria T cells was higher in patients with PI-IBS and post-infected controls compared to healthy volunteers. Moreover, no differences in T lymphocytes, mast cells or pro-inflammatory cytokines were detected between PI-IBS patients and infected control subjects three years after a Salmonella infection, although only PI-IBS patients were hypersensitive to rectal distention (14). Hence, the evidence supporting the hypothesis that chronic microscopic inflammation or immune activation is involved in PI-IBS is rather limited, confined to small patient numbers and mostly based on biopsies collected 3-4 months after the episode of IGE, and thus clearly deserves further study. Of note, the severity of the insult to the mucosa differs significantly between pathogens, most likely explaining why some studies still report inflammatory changes after 3-6 months and others do not. For example, Campylobacter jejuni produces a severe insult with mucosal ulceration while viruses rather produce a more transient inflammation without ulcerations. The risk to develop PI-IBS is not only determined by the nature of the pathogen, the severity of the infection, age and gender, but also by psychological factors (15). Anxiety, depression and fatigue are identified as risk factors for PI-IBS $(16 ; 17)$. Correspondingly, patients with a history of anxiety and depression have impaired recovery of PI-IBS during a six to eight year period of follow-up $(17 ; 18)$. How psychological factors may increase the risk to develop PIIBS however remains unclear. Of interest, it is becoming increasingly clear that brain-gut interactions may significantly modulate the intestinal immune system, and thus the immune response to a pathogen. Chronic psychological stress impairs the immune system by altering patterns of cytokine secretion, thereby increasing the vulnerability to infections $(19 ; 20)$. For example, chronic psychological stress in rats alters the immune response from antigen-induced oral tolerance to sensitization with a subsequent antigen-induced increase in inflammatory cells and mucosal permeability (21). Likewise, chronic stress alters the Th1/Th2 balance $(21 ; 22)$ and leads to an accumulation of myeloid-derived suppressor cells in the peripheral blood and bone marrow associated with immunosuppression (23). Moreover, chronic stress in mice activates the IL-10/STAT3 axis, thereby suppressing the production of pro-inflammatory cytokine IL-12 and inducing a shift of 
Th1/Th2 cytokines toward a Th2 predominance (24). Interestingly, a decreased Th1 immune response has recently been shown to lead to increased susceptibility of mice to infection with the enteropathogenic Escherichia coli (25). Based on these findings, we hypothesized that psychological factors may increase the risk to develop PI-IBS by modulation of the immune response towards the gastrointestinal infection.

In December 2010, two small villages in Belgium (Hemiksem and Schelle) suffered from a large community-wide multi-pathogen outbreak of gastroenteritis due to contamination of the tap water with water from a small creek (26). At least 18000 local residents were exposed to this contaminated water supply (26). In the present study, we aimed 1. to explore the potential relationship between psychological factors, the immune response in the acute infectious phase and the risk to develop infectious gastroenteritis and/or PI-IBS; and 2. to study in depth the potential role of persistent immune activation in those patients who developed PI-IBS. To this end, data were prospectively collected using questionnaires evaluating demographics and psychological data, exposure to tap water, bowel habits before and during the outbreak. Additionally, clinical symptoms and immune activation were assessed by validated questionnaires and quantification of cytokine expression in (stimulated) peripheral blood mononuclear cells, in plasma and in rectal biopsies respectively in the acute phase of the infection and after 1 year.

\section{MATERIAL AND METHODS:}

An epidemic outbreak of IGE occurred in Hemiksem and Schelle (Belgium) between December 6 and 9 in 2010 by contamination of the municipal tap water with local creek water as reported by the Local Health Authority (26). Although not officially established, the contamination was most likely caused by a misconnection of hoses by fireman during fire extinction. From December 6 onwards, the 18620 inhabitants of both villages were exposed to contaminated water before being alarmed at December 9 by the Local Health Authority. From December 9 onwards, the population was advised to stop drinking tap water. At December 10, a task force was set up in our unit to study the epidemiology and long-term outcome of the outbreak. The study protocol was approved by the Ethics Committee of the University Hospitals Leuven and all patients gave informed consent.

Study design:

In the acute phase (within 2 weeks), 603 subjects who consulted their general physician because of IGE were approached by phone. This subset of patients underwent a standardized interview, filled out questionnaires (details below) and was approached to provide blood samples for PBMC isolation, faecal samples and rectal biopsies. Based on questionnaires, data were collected on demographics 
(age, gender), exposure to tap water, bowel habits before and during the outbreak (ROME-III and GSRS questionnaires) and IGE characteristics, including onset of symptoms, duration of diarrhea, severity of abdominal discomfort and use of antibiotics. Moreover, to assess their psychological profile, subjects were asked to fill out the Hospital Anxiety and Depression Scale (HADS) based on how the subject felt 2 weeks before the outbreak and the Patient Health Questionnaire (PHQ-15) based on how the subject felt 4 weeks before the infection. Demographic data on the different recruitment strategies are reported in supplemental Table 3. To avoid the possibility that gastrointestinal symptoms at the time of infection influenced recall somatization scores, we modified the PHQ-15 by excluding three gastrointestinal items to create the PHQ-12 Somatic Symptom (PHQ-12 SS) scale (27). In addition, all households ( $n=7711)$ of the 2 affected villages were contacted by mailing within 3 months after the outbreak. Subjects were invited to participate in our study and to complete questionnaires as described above. Subjects were excluded when younger than 18 years, if they had pre-existing IBS, if symptoms of IGE started outside the 2 week period following the first day of drinking water contamination and if the Rome III questionnaire was incompletely answered.

One year after the outbreak, all subjects who participated previously were re-contacted to assess the development of PI-IBS and were asked to complete the following questionnaires: ROME-III, GSRS, HADS and PHQ-12. In addition, patients were asked to provide blood samples for PBMC isolation, faecal samples and rectal biopsies.

Outcome measure / definitions:

IGE was defined as a period of self-reported acute gastrointestinal discomfort within 2 weeks after tap water contamination. The severity of diarrhea was classified as follows (28): Classic IGE was defined as passage of three or more unformed stools per $24 \mathrm{~h}$ with at least one accompanying symptom (nausea, vomiting, abdominal cramps or pain, fever, blood in stools). Moderate IGE was defined as passage of one or two unformed stools with at least one additional symptom. Mild IGE was defined as passage of one or two unformed stools without additional symptoms. Controls (noIGE) were subjects who were exposed but did not report symptoms of IGE. The severity of abdominal cramping and abdominal pain was scored as none, mild, moderate or severe. IBS and the different IBS subtypes were diagnosed according to the ROME III criteria (http://www.romecriteria.org/ assets/pdf/19_RomellI_apA_885-898.pdf). Subjects who reported IBS symptoms or had been diagnosed with IBS prior to December 6th 2010 were excluded from the study. The incidence of dyspepsia-like symptoms was based on 5 GSRS questions, namely epigastric pain and discomfort, sucking sensation in the epigastrium, nausea, bloating and regurgitation. A subject was considered as dyspeptic when the summated score on these questions is larger than 4. Finally, a total HADS anxiety 
or depression score from 0 to 7 was considered normal, 8 to 10 as borderline while scores above 10 were considered to be abnormal $(29 ; 30)$.

Statistical analysis, gene expression studies, PBMC immune-phenotyping and cytokine analysis in plasma and in supernatants of stimulated PBMCs are described in supplementary Material and Methods.

RESULTS:

Characterization of patient cohort:

A. Early phase

Patient cohort

Between December $6^{\text {th }}$ and $9^{\text {th }} 2010,18620$ residents in 7711 households in Hemiksem and Schelle were exposed to contaminated tap water and asked to participate in our study. 1379 subjects completed the questionnaires of which 411 individuals were excluded (Flowchart Fig. 1).

Of the 968 eligible subjects, 271 (28\%) reported IGE: 62\% presented with classic IGE, 19\% with moderate IGE and $19 \%$ with abdominal symptoms but without diarrhea (IGE w/o diarrhea). The number of IGE cases followed a steep incline from December 6 to $8^{\text {th }}$ and peaked on December $8^{\text {th }}$ (Fig. 2). Other main IGE symptoms included nausea (reported by $54 \%$ ), vomiting (61\%), abdominal cramping (55\%) and abdominal pain (69\%). The mean duration of illness was $7.7 \pm 8.2$ days, the mean duration of diarrhea was $5.2 \pm 8.4$ days. Nine out of 270 individuals ( $3 \%, n=1$ missing data on rectal bleeding) reported rectal blood loss.

Fifty six fecal samples were collected from subjects clinically diagnosed with IGE of which 26 specimens tested positive for an infectious pathogenic agent. Norovirus NoV GI.4, GII.4, GII.14, GII.7 was most frequently detected (15/56), followed by Giardia lamblia (5/56), Campylobacter ssp. $(4 / 56)$, adenovirus (1/56) and rotavirus A (1/56). Two patients were positive for more than 1 infectious agent: 1 with NoV GI.4 and GII.4, and 1 with Campylobacter jejuni and NoV GI.4 and GII.7.

Risk factors underlying the susceptibility to develop IGE

Participants were asked to provide information on their consumption and use of the drinking water. $39 \%$ of inhabitants drank tap water with an average of 3.7 glasses per day while almost all subjects used tap water to brush their teeth (98\%) or to wash vegetables and fruit (97\%).

As previous studies indicated psychological factors as risk factor to develop PI-IBS, subjects were asked to complete the HADS and PHQ-12 questionnaires to assess anxiety, depression and somatization 2 and 4 weeks before the outbreak respectively. As shown in Fig. 3A, the proportion of subjects with abnormal anxiety scores $(>10)$ was increased in the IGE group $(11 \%)$ compared to the exposed group (4\%, p $\chi 2=0.0001$ ). Also PHQ-12 somatization scores were increased in the IGE group 
(median=3 IQR[2-7]) compared to the exposed group (median=2.5 IQR[1-5], Mann Whitney-U test $p=0.0001$ ). The proportion of subjects with abnormal depression scores was not different between the IGE (4\%) and the exposed group (5\%) (Fig. 3A). Of note, the prevalence of pre-existing dyspepsialike symptoms before the IGE outbreak was higher in the IGE group compared to the exposed group ( $35 \%$ vs $19 \%$ respectively; $\mathrm{p} \times 2<0.0001$ ).

As shown in Table 1, univariate analyses revealed that the development of IGE was associated with gender, age, pre-existing dyspepsia-like symptoms and drinking contaminated tap water. Of interest, anxiety and PHQ12 somatization, but not depression scores, were significantly associated with the development of IGE (Table 1, Fig. 3A). Inclusion of the significant parameters in a multivariate binary logistic regression model identified age, pre-existing dyspepsia-like symptoms, drinking tap water and anxiety as independent risk factors to develop IGE (Table 1).

Of interest, analysis of the risk factors to develop IGE based on only individuals with classic IGE ( $n=168)$ compared to the IGE- group ( $n=697$ ) revealed very similar results (data not shown).

Table 1. Risk factors to develop IGE

\begin{tabular}{|c|c|c|c|c|c|c|c|}
\hline & Total & IGE+ & IGE- & $\begin{array}{c}\text { Univariate } \\
\text { Pearson } \chi^{2} \\
\text { p }\end{array}$ & OR $[95 \% \mathrm{Cl}]$ & $\begin{array}{c}\text { Multiva } \\
\text { riate } \\
\text { logistic } \\
\text { regressi } \\
\text { on } p\end{array}$ & OR $[95 \% \mathrm{Cl}]$ \\
\hline Acute phase & 968 & 271 & 697 & & & & \\
\hline Female, N (\%) & $496(51 \%)$ & $156(59 \%)$ & $340(49 \%)$ & 0.015 & & NS & \\
\hline Age, mean(SD) & $52(16)$ & $49(16)$ & $53(16)$ & $<0.0001 \#$ & $0.98[0.98-0.99]$ & 0.001 & $0.98[0.97-0.99]$ \\
\hline $\begin{array}{l}\text { Pre-existing dyspepsia-like } \\
\text { symptoms, N (\%) }\end{array}$ & $213(22 \%)$ & $93(35 \%)$ & $120(19 \%)$ & $<0.0001$ & & 0.026 & $1.1[1.0-1.1]$ \\
\hline \multicolumn{8}{|l|}{ Exposure to tap water: } \\
\hline Not reported, N (\%) & & $87(32 \%)$ & $2(0.3 \%)$ & & & & \\
\hline Drinking tap water, N (\%) & & $104(57 \%)$ & $242(35 \%)$ & $<0.0001$ & & $<0.0001$ & $2.6[1.8-3.7]$ \\
\hline \multicolumn{8}{|l|}{ Psychological factors: } \\
\hline $\begin{array}{l}\text { anxiety HADS score (mean, } \\
\qquad 95 \% \mathrm{Cl}]\end{array}$ & & $\begin{array}{c}5.29[4.78- \\
5.79]\end{array}$ & $\begin{array}{c}3.99[3.73- \\
4.25]\end{array}$ & $<0.0001 \#$ & $1.1[1.1-1.2]$ & \multirow[t]{2}{*}{0.010} & \multirow[t]{3}{*}{$1.1[1.0-1.1]$} \\
\hline $\begin{array}{l}\text { depression HADS score } \\
\text { (mean, 95\%Cl] }\end{array}$ & & $\begin{array}{c}3.37[2.95- \\
3.80]\end{array}$ & $\begin{array}{c}3.19[2.93- \\
3.45]\end{array}$ & 0.62 & $1.0[0.97-1.05]$ & & \\
\hline $\begin{array}{l}\text { somatization score (PHQ-12) } \\
\text { (mean, } 95 \% \mathrm{Cl}]\end{array}$ & & $\begin{array}{c}4.50[4.05- \\
4.95]\end{array}$ & $\begin{array}{c}3.37[3.13- \\
3.62]\end{array}$ & $<0.0001 \#$ & $1.1[1.06-1.14]$ & NS & \\
\hline
\end{tabular}


Immune response in the infectious period and relationship with psychological factors

To evaluate the relationship between psychological factors and the immune response to the infection, PBMCs were immunologically phenotyped in great detail, plasma cytokines were measured and the expression of inflammatory genes was studied in rectal biopsies. Plasma cytokine analysis of

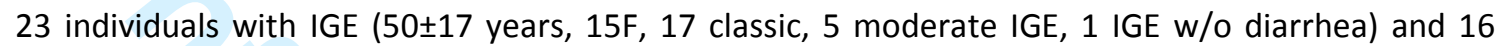
healthy controls ( $46 \pm 15$ years, 9F) revealed no differences in cytokine levels for IFNY, IL4, IL13, IL6, IL8, IL10, IL13 or IL17 (sFig.1). In contrast, inflammatory gene mRNA expression for IL10, TNF $\alpha$, IL6, MCP-1 and IFN $\gamma$ was significantly upregulated in rectal biopsies of 9 subjects with IGE (mean age $44 \pm 16$ years, 5F) compared to 9 healthy controls (mean age $54 \pm 17$ years, 5F) (Fig. 3C). mRNA expression levels for IL4 and IL5 were below detection limit.

Next, we evaluated the potential relationship between psychological factors and the immune response to the infectious pathogens in the total IGE group. The number of CD4+CD8-IL2+ T cells, a cell type critical in maintaining regulatory $T$ cells (Treg)(31), was inversely correlated with anxiety, depression and somatization (Fig. 3B). The observed significances for CD4+CD8-IL2+ T cells did not survive FRD correction for multiple testing. There was no correlation between psychological scores and Tregs, Th1, Th2 or Th17 cells (data not shown). 
B. One year follow-up

Incidence of IBS and type of gastrointestinal symptoms:

567 of the 968 (59\%) participants returned the questionnaires 1 year after the outbreak, including $180 / 271$ (66\%) of the IGE group and 387/697 (56\%) of the exposed subjects who did not develop IGE (Fig. 1). 29 subjects (8 IGE, 21 exposed) were excluded because of incomplete Rome questionnaires (Fig. 1). 19.8\% (34/172) of IGE subjects developed IBS (IGE+IBS+, OR: 3.0[1.8-4.9]) compared to $6.6 \%$ (24/366) of the exposed group that did not develop IGE (IGE-IBS+, pX2<0.0001). Of the PI-IBS patients, $24 \%$ had IBS-C, 35\% IBS-D, 6\% IBS-M and 35\% IBS-U. The main gastrointestinal symptoms, assessed using the GSRS questionnaire, are shown in Supplemental Figure 2. Notably, IGE patients also developed more dyspeptic symptoms since the outbreak; $25 \%$ of IGE patients developed an abnormal dyspepsia-like symptom score compared to $14 \%$ in the exposed no-IGE group (px2<0.003, OR: 2.0 [1.3-3.3]). Demographic data on subjects who responded versus who did not at 1 year follow-up are reported in supplemental Table 4.

\section{Psychological factors and immune profile 1 year after the outbreak}

The proportion of subjects with an abnormal anxiety score was higher in the IGE subjects whether they did (IGE+IBS+12\%) or did not develop IBS (IGE+IBS-, 9\%) compared to the exposed group and in subjects that developed IBS but had no IGE (IGE-IBS+, 25\%) compared to the group that did not develop IGE or IBS (exposed IBS-, 3\%, p X2<0.0001, Fig. 4A). PHQ-12 somatization scores were also increased in the PI-IBS- (median: 6 IQR[5-7]), PI-IBS+ (median: 5 IQR[5-6]) and exposed IBS+ (median=5.5 IQR[5-6]) groups compared to the exposed IBS- group (median=3 IQR[1-5], p Kruskal Wallis with Dunns' correction for multiple comparisons) (Fig. 4A). In contrast, the proportion of subjects with abnormal depression scores was similar amongst groups (Fig. 4A).

To evaluate the role of persistent immune activation in the development of PI-IBS, PBMC phenotypes and plasma cytokine levels were assessed in 108 individuals (for details, see Supplemental Table 2), including 17 PI-IBS+. CD4-CD19+ B cells were increased in IGE+IBS+ compared to exposed IBS- individuals (Fig. $4 \mathrm{~B}$ ). However, there were no differences in B cell subpopulations (sFig. 2), immuno-phenotypes of T cell populations, DCs or NK cells when comparing IGE+IBS+ with IGE+IBS- and IGE-IBS- subjects. When assessing cytokine levels in supernatants of stimulated PBMCs ( $n=77$, supplemental Table 2), IL10 and IL-8 levels were increased in PI-IBS compared to the exposed group while IL13, IL1b and TNF $\alpha$ levels were similar in all groups (Fig. 4C). Plasma cytokine levels (IFNg, IL17, IL10, IL4, IL6, IL8 and IL13 were not different between IGE+IBS+ and IGE+IBS- or exposed groups (sFig. 4). None of the results could withstand Bonferroni multiple testing correction. 
Finally, inflammatory cytokines mRNA was assessed in rectal mucosal biopsies of 32 subjects, including 9 IGE+IBS-, 5 IGE+IBS+, 15 IGE-IBS- and 3 IGE-IBS+ (sTable 3). There was no difference in mRNA expression level of IL10, IL6, IL8, TNFa nor hMCP-1 between IGE+IBS+, IGE+IBS- and exposed groups (sFig. 5). IFN $\gamma$ and IL5 mRNA expression levels were below detection limits ( $\mathrm{Ct}=35$ ).

\section{Risk factors to develop PI-IBS}

As shown in Table 2, univariate analysis revealed that the development of PI-IBS after IGE is associated with anxiety, somatization and GSRS dyspepsia-like symptom scores, all prior to outbreak and at follow-up, together with age and IGE severity (number of diarrhea days, abdominal cramping).

Table 2. Risk factors to develop PI-IBS after IGE

\begin{tabular}{|c|c|c|c|c|c|}
\hline & Total & $\begin{array}{c}\text { Self-reported } \\
\text { IGE }\end{array}$ & $\begin{array}{c}\text { Exposed, no } \\
\text { IGE }\end{array}$ & $\begin{array}{l}\text { Pearson } \chi^{2} p \text { IGE } \\
\text { vs no IGE }\end{array}$ & \\
\hline 1 year follow-up & 538 & 172 & 366 & & \\
\hline Female, $\mathrm{N}(\%)$ & 258 (48\%) & $92(53 \%)$ & $166(45 \%)$ & 0.08 & \\
\hline Age, mean (SD) & $52(15)$ & $50(15)$ & $54(15)$ & $0.016 \#$ & \\
\hline \multirow[t]{2}{*}{ IBS, N (\%) } & $58(11 \%)$ & $34(20 \%)$ & $24(7 \%)$ & $<0.0001$ & \\
\hline & PI-IBS & IGE & $\begin{array}{c}\text { Univariate } \\
\text { binary logistic } \\
\text { regression test } \\
\mathbf{p}\end{array}$ & $\begin{array}{l}\text { Multivariate } \\
\text { logistic } \\
\text { regression p }\end{array}$ & OR $[95 \% \mathrm{Cl}]$ \\
\hline \multicolumn{6}{|l|}{ Risk factors PI-IBS acute phase } \\
\hline Age, mean(SD) & $42.4(13.2)$ & $52.0(15.3)$ & 0.002 & 0.010 & $0.95[0.91-0.99]$ \\
\hline $\begin{array}{l}\text { Number of diarrhea days, mean } \\
\text { (SD) }\end{array}$ & $7.8(7.4)$ & $4.7(5.4)$ & 0.03 & NS & \\
\hline Abdominal cramping & & & 0.017 & NS & \\
\hline HADS anxiety & & & 0.014 & NS & \\
\hline PHQ12 somatization & & & 0.0001 & 0.008 & $1.16[1.03-1.31]$ \\
\hline $\begin{array}{l}\text { Pre-existing dyspepsia-like } \\
\text { symptoms }\end{array}$ & & & 0.013 & NS & \\
\hline \multicolumn{6}{|l|}{$\begin{array}{l}\text { Risk factors PI-IBS at } 1 \mathrm{yr} \\
\text { follow-up }\end{array}$} \\
\hline HADS anxiety & & & 0.044 & NS & \\
\hline PHQ12 somatization & & & $<0.0001$ & NS & \\
\hline Dyspepsia-like symptoms & & & $<0.0001$ & 0.0001 & $1.17[1.05-1.30]$ \\
\hline
\end{tabular}

\# $\mathrm{p}$ binary logistic regression test, NS: not significant in multivariate analysis 
In contrast, the development of PI-IBS was not associated with gender, other IGE characteristics, drinking tap water or number of glasses water consumed per day, use of medication (antibiotics, anti-diarrhea, ORS), allergy (hay fever, eczema), asthma, atopic constitution, alcohol, smoking, drugs, or familial history of IBD/IBS, prior major depression or traumatic life time experience, depression at time of outbreak or at follow-up. The multivariate logistic regression model at the time of infection revealed age and somatization as risk factor to develop PI-IBS. Inclusion of the significant parameters at 1 year follow-up identified the presence of dyspepsia-like symptoms as independent factor associated with PI-IBS (Table 2).

To study the relative contribution of psychological factors and IGE as risk factors to develop IBS, we performed an extra analysis on all subgroups (IGE-IBS-, IGE-IBS+, IGE+IBS- and IGE+IBS+). In line with our findings above, anxiety before the outbreak predicts IGE development $(p=0.001$, path $b$ in Fig. 5A) and IBS at follow-up ( $p<0.0001$, Fig. 5B) directly. When IGE status was added to the model in Fig. $5 B$, both anxiety $(p<0.0001$, path a' in Fig. $5 A)$ and IGE $(p=0.0018$, path $c$ in Fig. $5 A)$ were identified as predictors of IBS at follow-up, without the direct effect of anxiety being changed compared to the model in Fig. 5B. Therefore, the effect of anxiety on IBS at follow-up is not only direct but also to a limited though significant extent indirect through increasing vulnerability to IGE development. Similarly, somatization scores before the outbreak predicted IGE $(p=0.0088$, path $b$ in Fig. $5 C$ ) and IBS ( $p<0.0001$, path a in Fig. 5D). In combination, both somatization ( $p<0.0001$, path $a^{\prime}$ in Fig. 5C) and IGE (path c in Fig. 5C) are significant predictors of IBS at follow-up, without the effect of somatization being significantly changed compared to the model in Fig. 5D. This indicates that the effect of somatization on IBS is not only direct, but also to a certain extent indirect through increased risk of IGE during the outbreak.

Finally, depression before the outbreak predicts IBS ( $p=0.0038$, path a in Fig. $5 F$ ) but not the development of IGE ( $p=0.15$, path $b$ in Fig. $5 E$ ). When IGE status was added to the model, both depression ( $p=0.0077$, path $a^{\prime}$ in Fig. $5 E$ ) and IGE ( $p=0.0002$, path $c$ in Fig. $5 E$ ) were predictors of IBS at follow-up, without the effect of depression being changed compared to $F$, suggesting that the effects of depression and IGE on the development of IBS are independent of each other.

To evaluate if the acute immune response during the episode of IGE predicts the development of PI-IBS, the phenotype of the PBMCS of PI-IBS patients in the acute phase was compared with those who did not develop PI-IBS. Although the $n$-values are small, increased numbers of Th2 cells were observed at the time of IGE in individuals who went on to develop PI-IBS compared to those who did not ( $p=0.02$, Mann Whitney $U$ t-test, Fig. 6$)$. There is also a trend towards decreased numbers of Th1 cells and CD4+CD8-IL2+ cells $(p=0.07)$ during IGE in subjects who develop 
PI-IBS 1 year later compared to those who do not (Fig. 6). Plasma cytokine levels (IFNg, IL17, IL10, IL4, IL6, IL8 and IL13) were not different (data not shown).

\section{DISCUSSION}

A large cohort exposed to contaminated drinking water was prospectively studied to assess the risk to develop PI-IBS, focusing on the role of psychological factors and the immune response both in the acute phase and 1 year after the outbreak. Our study indicates that IGE and psychological factors (anxiety and somatization but not depression) are associated with an increased risk to develop PI-IBS. Of interest though, we showed that anxiety and somatization are also risk factors to develop IGE, thereby indirectly adding to the increased risk to develop IBS. Thorough analysis of the immune response in the acute IGE phase revealed that anxiety was associated with a reduction in IL2 expressing $C D 4+C D 8-T$ cells, suggesting a link between psychological factors, immune response and the observed increased risk to develop IGE. Moreover, we found that patients who developed PI-IBS had a lower number of CD4+CD8- IL2+ T cells and Th1 cells but an increased number of Th2 cells at the time of infection. These data suggest that a predominant Th2 response against the infectious insult may predispose to progression to PI-IBS. The resolution of the systemic Th2 bias by one year follow-up is consistent with a shift in pathophysiology to the mucosa, and may explain why cohort studies have found conflicting results when determining whether IBS patients have a systemic Th2 bias $(14 ; 32 ; 33)$. Based on these findings, we propose that psychological factors, in particular anxiety and somatization, increase the risk to develop PI-IBS. This effect is to a certain extent explained by increasing the susceptibility to develop IGE, possibly by skewing the immune response towards a predominant Th2 response at the time of infection.

An episode of infectious gastroenteritis is a well-defined risk factor to develop IBS (34). Here, we report on a large cohort of more than 18000 residents exposed to drinking water contaminated by creek water containing Norovirus, Campylobacter spp and Giardia lamblia. Of the 968 residents who participated, $28 \%$ developed symptoms of IGE. Similar to previous studies (8), IGE was associated with an increased risk (odds ratio of 3 ) to develop PI-IBS, i.e. $20 \%$ of IGE patients was identified as IBS compared to $7 \%$ in the exposed control group. This proportion is higher compared to the $8 \%$ reported following a food-borne outbreak with Salmonella (3), but lower than the $36 \%$ detected in the mixed waterborne outbreak of Walkerton (9). The proportion of IBS-C ( $35 \%$ in the PIIBS group compared to less than $10 \%$ in literature) tends to be higher compared to previous studies $(35 ; 36)$. The explanation for this difference remains speculative but the type and localization of infection may be factors that determine the clinical phenotype. In addition to severity of IGE and age, and consistent with previous studies (2;12), psychological factors, in particular anxiety and 
somatization, were identified as risk factors to develop IBS (37) and PI-IBS (2;12). A novel and intriguing finding, however, is that anxiety and somatization are also associated with an increased susceptibility to develop IGE. This observation suggests that the increased risk of subjects with increased anxiety and somatization to develop PI-IBS is partly explained by the observation that they are more susceptible for a gastrointestinal infection. To what extent this results from stress-induced impaired barrier function (38) or impaired immune function with hampered clearance of the infectious agent (25) is hard to investigate. Of note, age is protective against IGE and this may possibly be directly related to age or may be due to previous exposure. Moreover, at the time of infection, anxiety and depression were inversely correlated with the percentage of CD4+CD8- IL-2producing T cells in peripheral blood. In line, subjects with IGE who developed PI-IBS also had low numbers of CD4+CD8- IL-2+ T cells at the time of infection. Moreover, these individuals revealed a more Th2 skewed immune response during the infectious challenge, as evidence by increased Th2 cells and reduced Th1 cells in the blood samples collected during the period of IGE. As IL-2 is an important Th1 cytokine and CD4+CD8-IL2+ T cells are involved in regulatory $T$ cell biology and immune homeostasis (31), the reduction in CD4+IL-2+ T cells related to anxiety and depression may theoretically contribute to the increased susceptibility for infection and perhaps set the stage to develop PI-IBS. It should be emphasized though that the number of blood samples studied is low, and that this conclusion thus remains very speculative. Nevertheless, animal studies support the notion that psychological factors can drive the Th1/Th2 balance towards Th2 predominance (24), and thereby increase susceptibility to infection with enteropathogens (25).

Persistent mucosal microscopic inflammation with increased mast cells, lamina propria T cells and enterochromaffin cells has been demonstrated in intestinal biopsies collected from PI-IBS patients (2;13;39). In addition, Gwee et al. (13) showed increased IL-1beta expression in PI-IBS rectal biopsies taken 3 months after the infection. However, we failed to detect any changes in expression of pro-inflammatory cytokines in rectal biopsies of collected 1 year after infection. Similar findings were reported by Mearin et al (14) although it has to be emphasized that patients were studied three years after gastroenteritis. Our results may be explained by the fact that the infectious pathogens involved (noroviruses, Giardia lamblia and Campylobacter spp) do not infect the distal gut and thus may not induce long-term immunological changes in the rectum. However, we did find upregulation of pro-inflammatory cytokines in the acute infectious phase arguing against this possibility. Moreover, we recently found that submucosal neurons of rectal biopsies of IGE+IBS patients from this cohort were more sensitive to the TRPV1 agonist capsaicin compared to biopsies of the IGE+IBSgroup (40). In line, Mearin et al. showed hypersensitivity to rectal distention in PI-IBS patients compared to infected patients who did not develop IBS, even though no differences were found in 
rectal biopsies. Taken together, these data indicate that intestinal sensory function can indeed be affected by an episode of IGE, even in the absence of local persistent inflammation.

To further evaluate the role of immune activation in PI-IBS, we also thoroughly phenotyped PBMCs and quantified plasma cytokine levels. Of interest, we found increased CD4-CD19+ B cell numbers in PI-IBS+ compared to PI-IBS- patients. Our finding that PI-IBS patients have a Th2 predominant immune response at the time of infection, is compatible with the assumption that a humoral immune response could be involved in PI-IBS. This hypothesis is indirectly supported by 2 recent studies in IBS patients showing increased activation of mucosal B lymphocytes and plasma cells in jejunal biopsies (41) and increased activation level of blood B cells (42). Similar to previous studies in IBS patients $(43 ; 44)$ we also found an increase in serum IL-8 levels, however, no other signs of aberrant immune activation in PI-IBS were observed.

The major strength of our study is that we prospectively studied a community sample of exposed and infected subjects and assessed psychological and immunological factors in great detail both in the acute infectious phase and 1 year later. This allowed us to identify psychological factors not only as a risk factor to develop PI-IBS but also to unravel its impact on the susceptibility to develop IGE. Moreover, this is the first study reporting on the immune response during the period of IGE, albeit in a small group of subjects, and to relate this to the risk to develop PI-IBS 1 year later. The major weakness of the study is the relatively low sample size of rectal biopsies and blood samples, in particular in the acute infectious phase. As a result, some of our data do not withstand correction for multiple testing, and should be interpreted cautiously. Also, the low recruitment number may introduce some bias and therefore extrapolation of our data to a wider population should be done with caution. This low sample number is inherent to the fact that it is a prospective study and it is logistically difficult to recruit individuals during the acute period of the outbreak. Moreover, our study is limited by the fact that questionnaires on anxiety, depression and somatization before the infection were assessed in the acute infectious phase and we cannot exclude a recall bias due to gastrointestinal symptoms experienced due to the infection. In an attempt to circumvent this problem, we excluded questionnaires on gastrointestinal symptoms from the PHQ15 questionnaire.

Altogether, our study shows that the increased risk of patients with psychological comorbidity to develop PI-IBS is partly due to an increased susceptibility to develop IGE. Moreover, we provide evidence to speculate that subjects who develop a predominant Th2 response against the infectious agent are prone to develop a humoral immune response and subsequently PI-IBS. 


\section{CONFLICTS OF INTEREST}

The authors declare no conflict of interest

\section{AUTHOR CONTRIBUTIONS:}

All authors read and approved the final version of the manuscript.

Mira M Wouters: data acquisition, analysis and interpretation of data, writing and

\begin{tabular}{|c|c|}
\hline Anh Nguyen: & $\begin{array}{l}\text { critical revision of the manuscript for important intellectual content } \\
\text { data acquisition, analysis and interpretation of data }\end{array}$ \\
\hline Sander JM van Wanrooij: & $\begin{array}{l}\text { study concept and design, acquisition of data, analysis and } \\
\text { interpretation of data }\end{array}$ \\
\hline James Dooley: & data acquisition \\
\hline Javier Aguilera-Lizarra & data acquisition \\
\hline Winde Vanbrabant: & recruitment of study subjects, data acquisition \\
\hline Josselyn E Garcia-Perez: & data acquisition \\
\hline Lukas Van Oudenhove: & $\begin{array}{l}\text { statistical analysis, interpretation of data, critical revision of the } \\
\text { manuscript for important intellectual content }\end{array}$ \\
\hline Marc Van Ranst: & data acquisition \\
\hline Jan Verhaegen: & data acquisition \\
\hline Adrian Liston: & $\begin{array}{l}\text { study supervision, obtaining funding, critical revision of the } \\
\text { manuscript for important intellectual content }\end{array}$ \\
\hline uy Boeckxstaens: & $\begin{array}{l}\text { study supervision, obtaining funding, critical revision of the } \\
\text { manuscript for important intellectual content }\end{array}$ \\
\hline
\end{tabular}

FUNDING: GEB was funded by a governmental (Odysseus programme, G.0905.07) of the Research Foundation - Flanders (FWO) and university grant (Global Opportunities for Associations GOA 14.011). MMW is supported by a FWO postdoctoral fellowship. This work was funded by FWO research grant G.0699.10N to GB and MMW. AL received the ERC Start grant IMMUNO. TLAN is funded by VIB International PhD program.

TRIAL REGISTRATION: ClinicalTrials.gov ID: NCT01497847 entitled "Post-Infectious Irritable Bowel Syndrome (PI-IBS)" 


\section{FIGURE LEGENDS}

Figure 1. Flow chart of outbreak cohort

Figure 2. Incidence of gastroenteritis

Individuals who developed IGE between December 6 and 19 were included in the study.

Figure 3. Prevalence of psychological conditions prior to infectious gastroenteritis and correlation with IL2 expressing T cells in acute infectious phase

Panel A: Psychological status 2 weeks before the outbreak was assessed by HADS-anxiety and depression questionnaires and the PHQ12 somatization questionnaire. Panel B: HADS-anxiety, depression and somatization were inversely correlated with IL2 expressing CD4+ cells. Panel C: Relative mRNA expression levels of inflammatory genes in rectal biopsies were increased in subjects with IGE compared to non-infected, healthy volunteers (HV; relative expression to HPRT1 housekeeping gene). Infected subjects suffered significantly more from increased anxiety and somatization compared to exposed subjects who would not develop symptoms of gastroenteritis (no IGE).

Figure 4. Psychological factors, PBMC immunophenotypes and cytokine analysis of stimulated PBMCs at 1 year follow-up

Panel A: HADS-anxiety and depression and PHQ12 somatization were assessed 1 year after the outbreak. Both IBS groups (IGE+IBS+ and IGE-IBS+) showed increased levels of anxiety and somatization compared to healthy individuals. Panel $B$ : T cell phenotypes and $B$ cell numbers were assessed and compared between subjects who developed IBS compared to subjects who did not. Only B cell numbers were significantly increased in PI-IBS patients compared to the exposed IBSsubjects. Panel C: Cytokine release of stimulated mononuclear leukocytes (PBMC) was assessed as a measure of their activation in vivo.

PI NS: post-infectious no symptoms, ${ }^{*} p<0.05 ;{ }^{* *} p<0.001 ;{ }^{* * *} p<0.0001$

Figure 5. The effect of psychological factors on the susceptibility to develop infectious gastroenteritis and IBS symptoms

The effect of anxiety, somatization and depression was assessed on the development of infectious gastroenteritis and IBS symptoms. Fig. A, C and $E$ show the indirect effect of psychological factors on the development of IBS symptoms via increasing the vulnerability to gastroenteritis. Fig. B, C and $F$ show the direct effect of psychological factors on the development of IBS symptoms.

Figure 6. Thelper cell analysis in the acute infectious phase comparing individuals who will develop PI-IBS compared to who will become symptom free

Infected individuals who will develop PI-IBS (PI-IBS+) show a tendency for Th2 skewing towards the infectious pathogens as shown by increased Th2 numbers and a tendency toward decreased Th1 and CD4+CD8- IL expressing cells compared to infected individuals who will become symptom free (PIIBS-). ${ }^{*} \mathrm{p}<0.05$, Mann Whitney U t-test 
(1) Spiller RC, Jenkins D, Thornley JP et al. Increased rectal mucosal enteroendocrine cells, T lymphocytes, and increased gut permeability following acute Campylobacter enteritis and in post-dysenteric irritable bowel syndrome. Gut 2000;47(6):804-11.

(2) Dunlop SP, Jenkins D, Neal KR et al. Relative importance of enterochromaffin cell hyperplasia, anxiety, and depression in postinfectious IBS. Gastroenterology 2003;125(6):1651-9.

(3) Mearin F, Perez-Oliveras M, Perello A et al. Dyspepsia and irritable bowel syndrome after a Salmonella gastroenteritis outbreak: one-year follow-up cohort study. Gastroenterology 2005;129(1):98-104.

(4) McKendrick MW, Read NW. Irritable bowel syndrome--post salmonella infection. J Infect 1994;29(1):1-3.

(5) Porter CK, Faix DJ, Shiau D et al. Postinfectious gastrointestinal disorders following norovirus outbreaks. Clin Infect Dis 2012;55(7):915-22.

(6) Zanini B, Ricci C, Bandera F et al. Incidence of post-infectious irritable bowel syndrome and functional intestinal disorders following a water-borne viral gastroenteritis outbreak. Am J Gastroenterol 2012;107(6):891-9.

(7) Wensaas KA, Langeland N, Hanevik K et al. Irritable bowel syndrome and chronic fatigue 3 years after acute giardiasis: historic cohort study. Gut 2012;61(2):214-9.

(8) Thabane M, Kottachchi DT, Marshall JK. Systematic review and meta-analysis: The incidence and prognosis of post-infectious irritable bowel syndrome. Aliment Pharmacol Ther 2007;26(4):535-44.

(9) Marshall JK, Thabane M, Garg AX et al. Incidence and epidemiology of irritable bowel syndrome after a large waterborne outbreak of bacterial dysentery. Gastroenterology 2006;131(2):445-50.

(10) Marshall JK, Thabane M, Garg AX et al. Eight year prognosis of postinfectious irritable bowel syndrome following waterborne bacterial dysentery. Gut 2010;59(5):605-11.

(11) Ford AC, Talley NJ. Mucosal inflammation as a potential etiological factor in irritable bowel syndrome: a systematic review. J Gastroenterol 2011;46(4):421-31.

(12) Gwee KA, Leong YL, Graham C et al. The role of psychological and biological factors in postinfective gut dysfunction. Gut 1999;44(3):400-6.

(13) Gwee KA, Collins SM, Read NW et al. Increased rectal mucosal expression of interleukin 1 beta in recently acquired post-infectious irritable bowel syndrome. Gut 2003;52(4):523-6. 
(14) Mearin F, Perello A, Balboa A et al. Pathogenic mechanisms of postinfectious functional gastrointestinal disorders: results 3 years after gastroenteritis. Scand J Gastroenterol 2009;44(10):1173-85.

(15) Spiller RC. Role of infection in irritable bowel syndrome. J Gastroenterol 2007;42 Suppl 17:41-7.

(16) Dunlop SP, Jenkins D, Neal KR et al. Relative importance of enterochromaffin cell hyperplasia, anxiety, and depression in postinfectious IBS. Gastroenterology 2003;125(6):1651-9.

(17) Marshall JK, Thabane M, Garg AX et al. Eight year prognosis of postinfectious irritable bowel syndrome following waterborne bacterial dysentery. Gut 2010;59(5):605-11.

(18) Neal KR, Barker L, Spiller RC. Prognosis in post-infective irritable bowel syndrome: a six year follow up study. Gut 2002;51(3):410-3.

(19) Chiappelli F, Manfrini E, Franceschi C et al. Brain Corticosteroid Receptors: Studies on the Mechanism, Function, and Neurotoxicity of Corticosteroid Action. Proceedings of a conference. Arlington, Virginia, March 2-5, 1994. Ann N Y Acad Sci 1994;746:1-499.

(20) Dragos D, Tanasescu MD. The effect of stress on the defense systems. J Med Life 2010;3(1):10-8.

(21) Yang PC, Jury J, Soderholm JD et al. Chronic Psychological Stress in Rats Induces Intestinal Sensitization to Luminal Antigens. Am J Pathol 2006;168(1):104-14.

(22) Shaashua L, Sominsky L, Levi B et al. In vivo suppression of plasma IL-12 levels by acute and chronic stress paradigms: potential mediating mechanisms and sex differences. Brain Behav Immun 2012;26(6):996-1005.

(23) Jin J, Wang X, Wang Q et al. Chronic psychological stress induces the accumulation of myeloid-derived suppressor cells in mice. PLOS ONE 2013;8(9):e74497.

(24) Hu D, Wan L, Chen M et al. Essential role of IL-10/STAT3 in chronic stress-induced immune suppression. Brain Behav Immun 2014;36:118-27.

(25) Sharma R, Kapila R, Dass G et al. Improvement in Th1/Th2 immune homeostasis, antioxidative status and resistance to pathogenic $E$. coli on consumption of probiotic Lactobacillus rhamnosus fermented milk in aging mice. Age (Dordr) 2014;36(4):9686.

(26) Braeye T, DE SK, Wollants E et al. A large community outbreak of gastroenteritis associated with consumption of drinking water contaminated by river water, Belgium, 2010. Epidemiol Infect 2014;1-9.

(27) Spiller RC, Humes DJ, Campbell E et al. The Patient Health Questionnaire 12 Somatic Symptom scale as a predictor of symptom severity and consulting behaviour in patients with irritable bowel syndrome and symptomatic diverticular disease. Aliment Pharmacol Ther 2010;32(6):811-20.

(28) von SF, Tornieporth N, Waiyaki P et al. Risk and aetiology of diarrhoea at various tourist destinations. Lancet 2000;356(9224):133-4. 
(29) Zigmond AS, Snaith RP. The hospital anxiety and depression scale. Acta Psychiatr Scand 1983;67(6):361-70.

(30) Bjelland I, Dahl AA, Haug TT et al. The validity of the Hospital Anxiety and Depression Scale. An updated literature review. J Psychosom Res 2002;52(2):69-77.

(31) Amado IF, Berges J, Luther RJ et al. IL-2 coordinates IL-2-producing and regulatory T cell interplay. J Exp Med 2013;210(12):2707-20.

(32) Hughes PA, Zola H, Penttila IA et al. Immune activation in irritable bowel syndrome: can neuroimmune interactions explain symptoms? Am J Gastroenterol 2013;108(7):1066-74.

(33) Liebregts T, Adam B, Bredack C et al. Immune activation in patients with irritable bowel syndrome. Gastroenterology 2007;132(3):913-20.

(34) Grover M, Camilleri M, Smith K et al. On the fiftieth anniversary. Postinfectious irritable bowel syndrome: mechanisms related to pathogens. Neurogastroenterol Motil 2014;26(2):156-67.

(35) Neal KR, Barker L, Spiller RC. Prognosis in post-infective irritable bowel syndrome: a six year follow up study. Gut 2002;51(3):410-3.

(36) Dunlop SP, Jenkins D, Spiller RC. Distinctive clinical, psychological, and histological features of postinfective irritable bowel syndrome. Am J Gastroenterol 2003;98(7):1578-83.

(37) Nicholl BI, Halder SL, Macfarlane GJ et al. Psychosocial risk markers for new onset irritable bowel syndrome--results of a large prospective population-based study. Pain 2008;137(1):147-55.

(38) Yang PC, Jury J, Soderholm JD et al. Chronic psychological stress in rats induces intestinal sensitization to luminal antigens. Am J Pathol 2006;168(1):104-14.

(39) Lee KJ, Kim YB, Kim JH et al. The alteration of enterochromaffin cell, mast cell, and lamina propria T lymphocyte numbers in irritable bowel syndrome and its relationship with psychological factors. J Gastroenterol Hepatol 2008;23(11):1689-94.

(40) Balemans, D., Alpizar, Y. A., Nasser, Y., Valdez-Morales, E. E., Moonen, A., Cirillo, C., Vanner, S. J., Talavera, K., Vanden Berghe, P., Wouters, M. M., and Boeckxstaens, G. E. Evidence for Histamine-Mediated Sensitization of TRPV1 Signaling in Sensory Neurons in Mice and IBS Patients. Gastroenterology. 2014. Ref Type: Abstract

(41) Vicario M, Gonzalez-Castro AM, Martinez C et al. Increased humoral immunity in the jejunum of diarrhoea-predominant irritable bowel syndrome associated with clinical manifestations. Gut 2014.

(42) Ohman L, Lindmark AC, Isaksson S et al. B-cell activation in patients with irritable bowel syndrome (IBS). Neurogastroenterol Motil 2009;21(6):644-50, e27.

(43) McKernan DP, Gaszner G, Quigley EM et al. Altered peripheral toll-like receptor responses in the irritable bowel syndrome. Aliment Pharmacol Ther 2011;33(9):1045-52. 
(44) Scully P, McKernan DP, Keohane J et al. Plasma cytokine profiles in females with irritable bowel syndrome and extra-intestinal co-morbidity. Am J Gastroenterol 2010;105(10):2235-

43. 


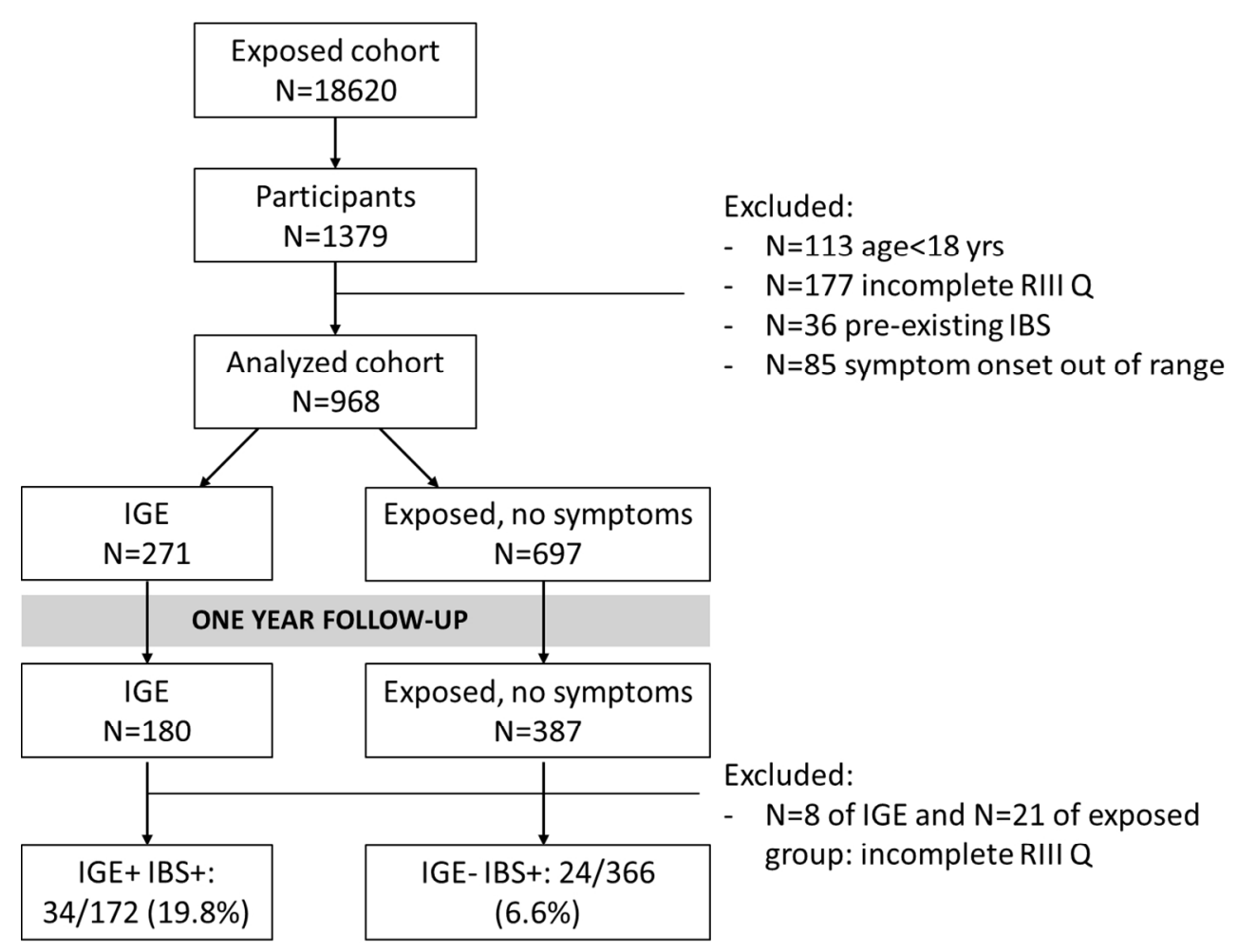

Flow chart of outbreak cohort $494 \times 377 \mathrm{~mm}(72 \times 72$ DPI $)$ 


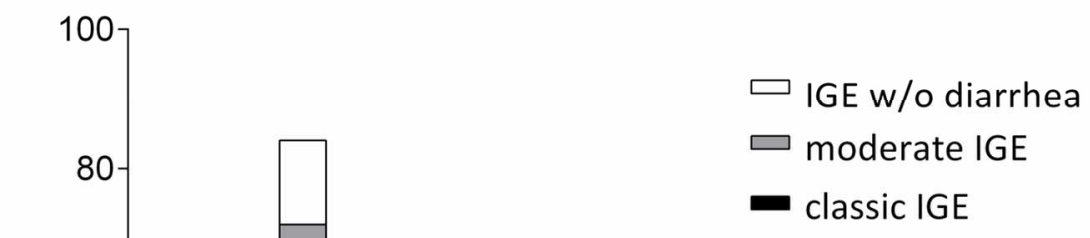

Incidence of gastroenteritis

Individuals who developed IGE between December 6 and 19 were included in the study.

$128 \times 87 \mathrm{~mm}(300 \times 300$ DPI $)$ 

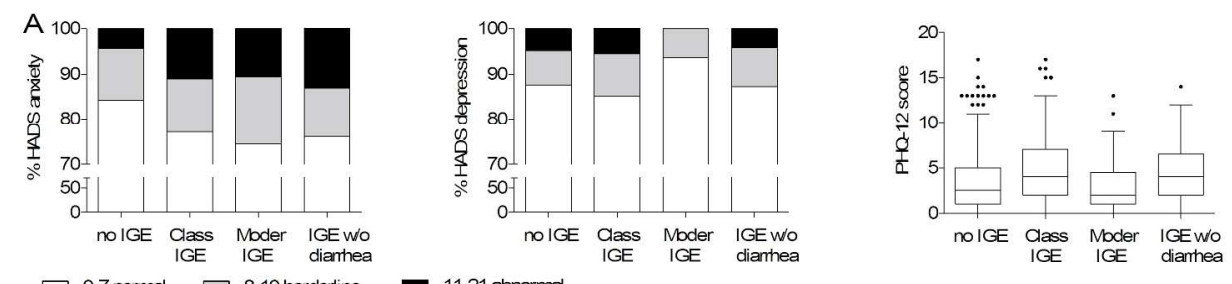

B
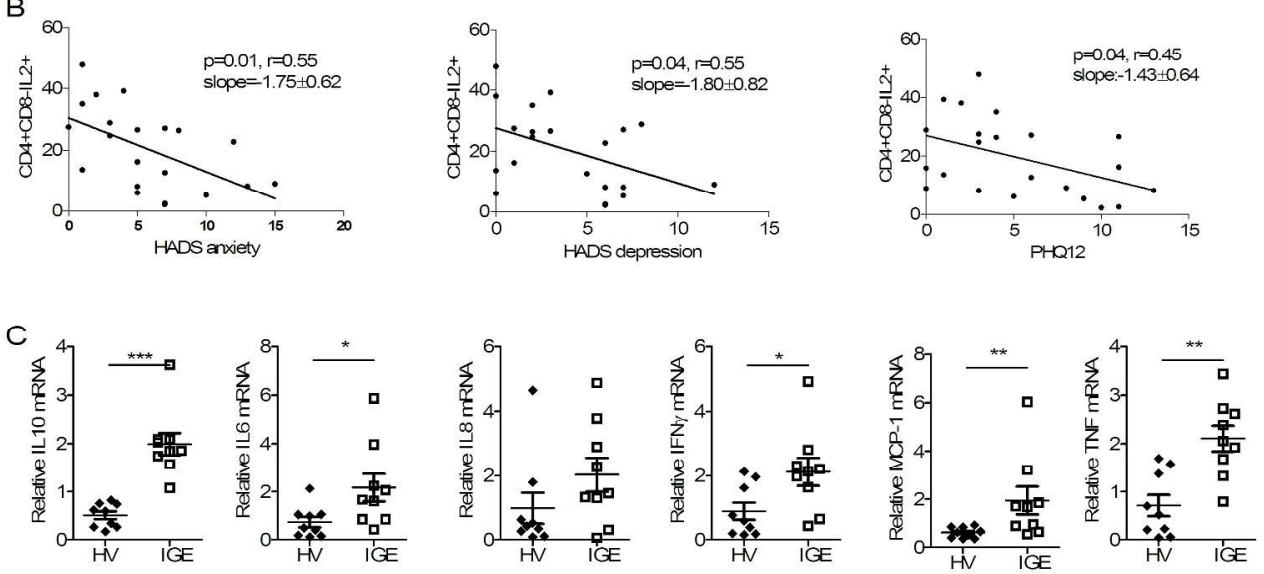

Prevalence of psychological conditions prior to infectious gastroenteritis and correlation with IL2 expressing $T$ cells in acute infectious phase

Panel A: Psychological status 2 weeks before the outbreak was assessed by HADS-anxiety and depression questionnaires and the PHQ12 somatization questionnaire. Panel B: HADS-anxiety, depression and somatization were inversely correlated with IL2 expressing CD4+ cells. Panel C: Relative mRNA expression levels of inflammatory genes in rectal biopsies were increased in subjects with IGE compared to noninfected, healthy volunteers (HV; relative expression to HPRT1 housekeeping gene). Infected subjects suffered significantly more from increased anxiety and somatization compared to exposed subjects who would not develop symptoms of gastroenteritis (no IGE).

\section{$276 \times 204 \mathrm{~mm}(300 \times 300$ DPI $)$}



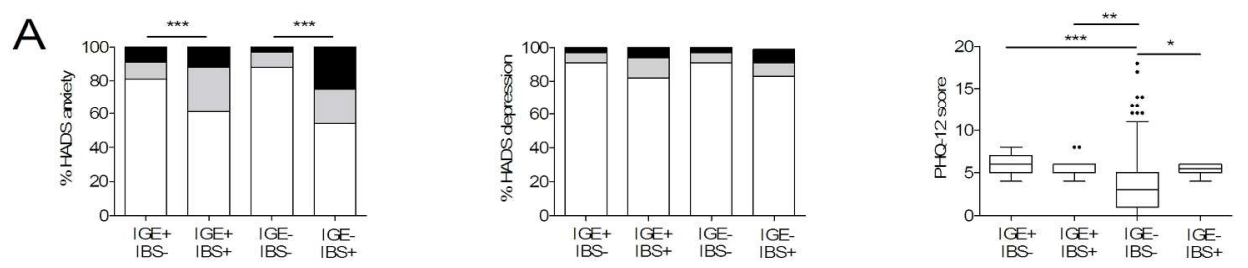

B
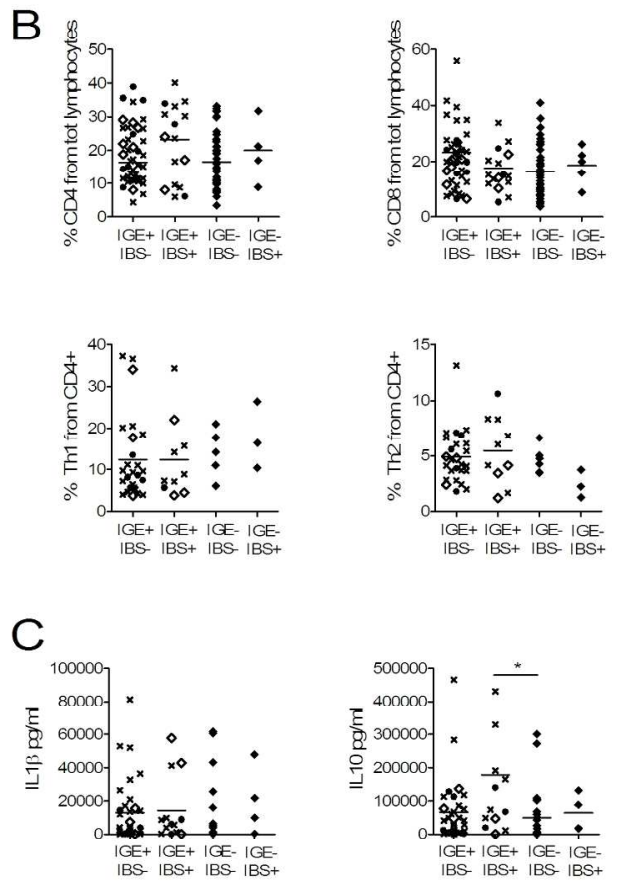

× classic IGE
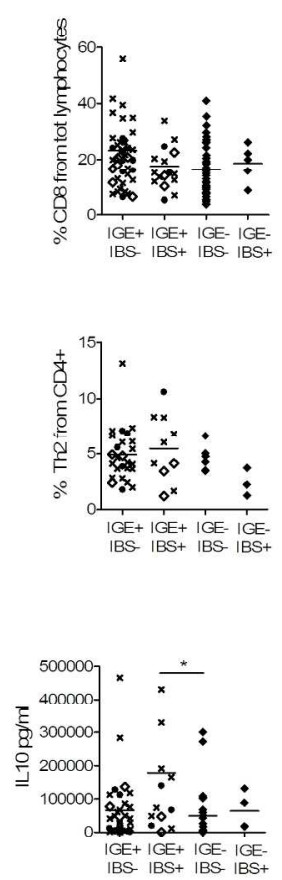
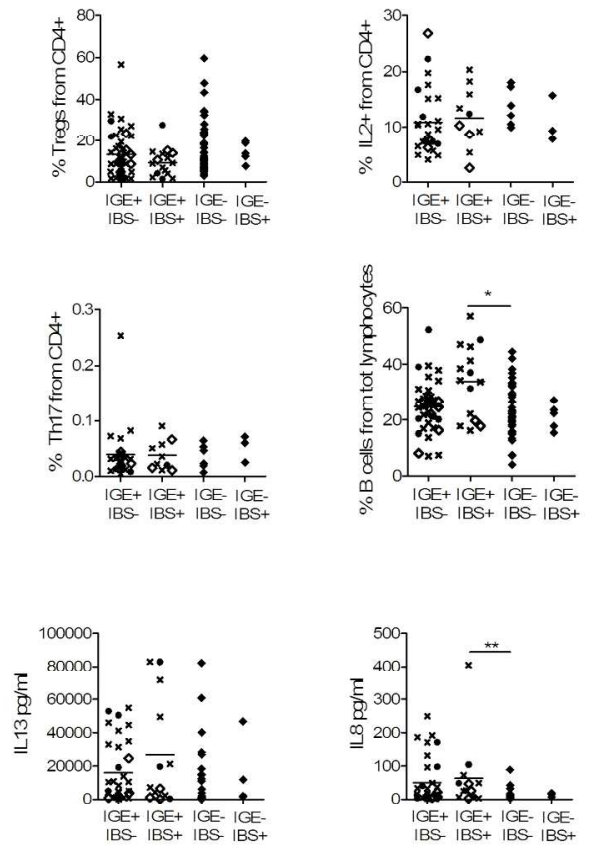

- IGE wo diarrhea

Psychological factors, PBMC immunophenotypes and cytokine analysis of stimulated PBMCs at 1 year followup

Panel A: HADS-anxiety and depression and PHQ12 somatization were assessed 1 year after the outbreak. Both IBS groups (IGE+IBS+ and IGE-IBS+) showed increased levels of anxiety and somatization compared to healthy individuals. Panel B: T cell phenotypes and B cell numbers were assessed and compared between subjects who developed IBS compared to subjects who did not. Only B cell numbers were significantly increased in PI-IBS patients compared to the exposed IBS- subjects. Panel C: Cytokine release of stimulated mononuclear leukocytes (PBMC) was assessed as a measure of their activation in vivo.

PI NS: post-infectious no symptoms, $* \mathrm{p}<0.05 ; * * \mathrm{p}<0.001 ; * * * \mathrm{p}<0.0001$

$$
197 \times 202 \mathrm{~mm}(300 \times 300 \text { DPI })
$$



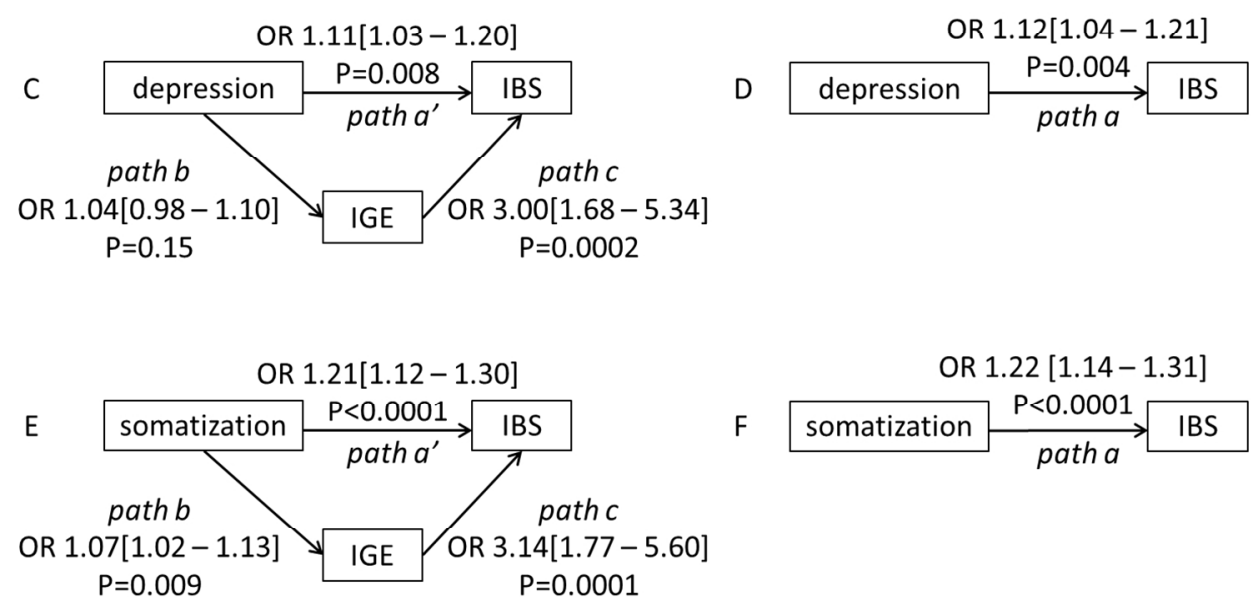

The effect of psychological factors on the susceptibility to develop infectious gastroenteritis and IBS symptoms

The effect of anxiety, somatization and depression was assessed on the development of infectious gastroenteritis and IBS symptoms. Fig. A, C and E show the indirect effect of psychological factors on the development of IBS symptoms via increasing the vulnerability to gastroenteritis. Fig. $B, C$ and $F$ show the direct effect of psychological factors on the development of IBS symptoms.

$546 \times 413 \mathrm{~mm}(72 \times 72 \mathrm{DPI})$ 

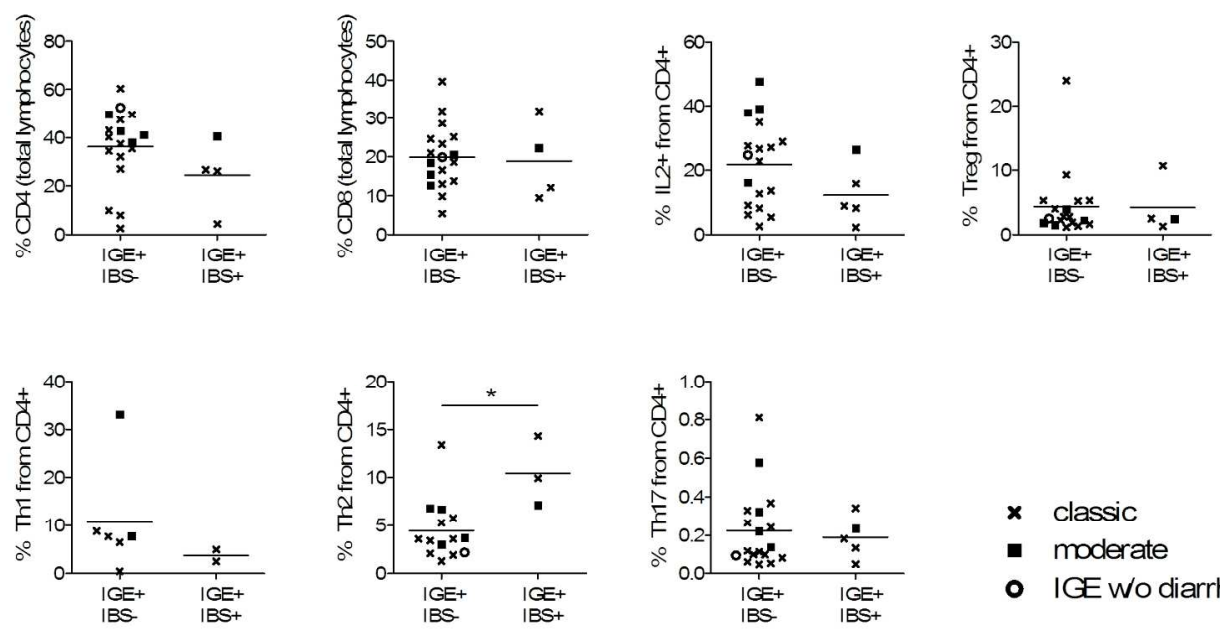

$\begin{array}{ll}\text { x } & \text { classic } \\ \text { - moderate } \\ \text { - IGE wo diarrhea }\end{array}$

T helper cell analysis in the acute infectious phase comparing individuals who will develop PI-IBS compared to who will become symptom free

Infected individuals who will develop PI-IBS (PI-IBS+) show a tendency for Th2 skewing towards the infectious pathogens as shown by increased Th2 numbers and a tendency toward decreased Th1 and CD4+CD8- IL expressing cells compared to infected individuals who will become symptom free (PI-IBS-). * $\mathrm{p}<0.05$, Mann Whitney U t-test

$197 \times 102 \mathrm{~mm}(300 \times 300 \mathrm{DPI})$ 
1

2

3

4

5

6

7

8

9

10

11

12

13

14

15

16

17

18

19

20

21

22

23

24

25

26

27

28

29

30

31

32

33

34

35

36

37

38

39

40

41

42

43

44

45

46

47

48

49

50

51

52

53

54

55

56

57

58

59

60

\section{Supplementary material and methods}

\section{Gene expression studies}

Total RNA was extracted by RNeasy Minikit (Qiagen Benelux, Antwerp, Belgium) and reverse transcribed by qScript cDNA Supermix (Quanta Science, Gaithersburg, MD, USA) according to the manufacturer's instructions. Real-time PCR was performed on a LightCycler ${ }^{\circledR} 96$ Real-Time PCR System (Roche Applied Science, Vilvoorde, Belgium) using the Lightcycler96 probes master mix as indicated by the manufacturer. Primer and probe sequences are described in supplemental Table 1. The relative expression of the target gene was calculated relative to the HPRT1 reference mRNA (1).

\section{Supplemental Table 1. Primer and probe sequences for quantitative PCR}

\begin{tabular}{|c|c|c|c|}
\hline Gene & Forward (5'-3' ) & Reverse (5'-3') & probe $\left(5^{\prime}-3^{\prime}\right)$ \\
\hline HPRT1 & TAAGATGAGAGTTCAAGTTG & GCAAATAAACAGTTTAGGAAT & TCCTATTGACATCGCCAGTAA \\
\hline$I F N Y$ & ACGAGATGACTTCGAAAA & TTCGCTTCCCTGTTTTAG & CGGTAACTGACTTGAATGTCCAACG \\
\hline IL5 & CAAGGACCTGCTGTATTC & $\begin{array}{c}\text { GCCTATTGTAGACAGTAAAATAAA } \\
\text { G }\end{array}$ & AATGAACAAGCAGGCACTTACAACA \\
\hline IL10 & AAGCCATGAGTGAGTTTG & GTCCTAGAGTCTATAGAGTCG & CGTATCTTCATTGTCATGTAGGCTTCT \\
\hline$T N F \alpha$ & GACGAACATCCAACCTTC & GGTGGTCTTGTTGCTTAA & ССССТGССССААТСССТTTA \\
\hline IL6 & TCTGGATTCAATGAGGAG & CTGGATCAGGACTTTTGTA & CTGGCTTGTTCCTCACTACTCTCA \\
\hline IL8 & AGAGGGCTGAGAATTCATAA & CACACAATACATGAAGTGTTGAA & CACTGGCATCTTCACTGATTCTTGG \\
\hline MCP1 & СCCCAGTCACCTGCTGTTAT & TCCTGAACCСACTTCTGCTT & ATTGTGGCCAAGGAGATCTG \\
\hline
\end{tabular}


Supplemental Table 2. Demographics of individuals who donated samples in 2012

\begin{tabular}{|c|c|c|c|c|}
\hline & PI-IBS- & PI-IBS+ & Exposed-IBS- & Exposed-IBS+ \\
\hline PBMC's and plasma & 42 & 17 & 44 & 5 \\
\hline Female, N (\%) & $22(52 \%)$ & $15(88 \%)$ & $20(45 \%)$ & $1(20 \%)$ \\
\hline Age, mean (SD) & $55(14)$ & $55(14)$ & $57(13)$ & $57(13)$ \\
\hline Classic diarrhea & 27 & 10 & & \\
\hline Moderate diarrhea & 8 & 3 & & \\
\hline IGE w/o diarrhea & 7 & 3 & & \\
\hline Unknown IGE type & & 1 & & \\
\hline $\begin{array}{c}\text { Stimulated PBMC } \\
\text { supernatants }\end{array}$ & 33 & 13 & 27 & 4 \\
\hline Female, N (\%) & $17(52 \%)$ & $12(92 \%)$ & $15(56 \%)$ & $1(25 \%)$ \\
\hline Age, mean (SD) & $55(15)$ & $55(15)$ & $55(15)$ & $55(15)$ \\
\hline Classic diarrhea & 24 & 7 & & \\
\hline Moderate diarrhea & 6 & 3 & & \\
\hline IGE w/o diarrhea & 3 & 3 & & \\
\hline Rectal biopsies & 9 & 5 & 15 & 3 \\
\hline Female, N (\%) & $3(30 \%)$ & $4(80 \%)$ & $5(30 \%)$ & $1(33 \%)$ \\
\hline Age, mean (SD) & $52(13)$ & $42(14)$ & $59(13)$ & $60(13)$ \\
\hline
\end{tabular}


Supplemental Table 3. Demographics of individuals included in acute infectious phase through GP versus survey

\begin{tabular}{|c|c|c|c|c|}
\hline & $\begin{array}{c}\text { IGE+ } \\
\text { Recruited via GP }\end{array}$ & IGE- & $\begin{array}{c}\text { IGE+ } \\
\text { By questionnaires }\end{array}$ & $\begin{array}{l}\text { P IGE+ GP vs } \\
\text { IGE+ survey }\end{array}$ \\
\hline $\mathrm{n}$ & 102 & 697 & 169 & \\
\hline Female gender $(\mathrm{N}, \%)$ & $62(61 \%)$ & $340(49 \%)$ & $94(56 \%)$ & $0.45 \#$ \\
\hline Age (years $\pm S D)$ & $52 \pm 17$ & $53 \pm 16$ & $48 \pm 15$ & $0.159 *$ \\
\hline $\begin{array}{l}\text { Number of diarrhea days, } \\
\text { mean (SD) }\end{array}$ & $5 \pm 5$ & & $5 \pm 10$ & $0.281 *$ \\
\hline $\begin{array}{l}\text { Number of days with } \\
\text { abdominal discomfort }\end{array}$ & $7 \pm 5$ & & $8 \pm 10$ & $0.0001 *$ \\
\hline Classic IGE, N(\%) & $74(73 \%)$ & & $94(56 \%)$ & $0.0066 \#$ \\
\hline Moderate IGE, N(\%) & $18(18 \%)$ & & $32(19 \%)$ & \\
\hline $\begin{array}{l}\text { IGE without diarrhea, } \\
\mathrm{N}(\%)\end{array}$ & $8(8 \%)$ & & $41(24 \%)$ & \\
\hline Missing data, $\mathrm{N}(\%)$ & $1(1 \%)$ & & $2(1 \%)$ & \\
\hline Blood in stool & $2(2 \%)$ & & $7(4 \%)$ & $0.40 \#$ \\
\hline Fever, N(\%) & $20(20 \%)$ & & $32(19 \%)$ & $0.88 \#$ \\
\hline Abnormal anxiety $(n, \%)$ & $10(10 \%)$ & $29(4 \%)$ & $19(11 \%)$ & $0.417 \#$ \\
\hline $\begin{array}{l}\text { Abnormal depression } \\
(n, \%)\end{array}$ & $6(6 \%)$ & $32(5 \%)$ & $5(3 \%)$ & $0.320 \#$ \\
\hline PHQ-12 (mean $\pm S D)$ & $5 \pm 4$ & $3 \pm 3$ & $4 \pm 4$ & $0.583 *$ \\
\hline $\begin{array}{l}\text { IBS (n IBS/n total subjects } \\
\text { at follow-up,\%) }\end{array}$ & $14 / 68(21 \%)$ & $24 / 365(7 \%)$ & $20 / 104(19 \%)$ & $0.827 \#$ \\
\hline
\end{tabular}

$\sharp$ P Fishers exact test, * Mann Whitney $U$ test 
1

2

3

4

5

6

7

8

9

10

11

12

13

14

15

16

17

18

19

20

21

22

23

24

25

26

27

28

29

30

31

32

33

34

35

36

37

38

39

40

41

42

43

44

45

46

47

48

49

50

51

52

53

54

55

56

57

58

59

60

Supplemental Table 4: Demographic data on subjects who responded versus who did not at 1 year followup

\begin{tabular}{lccc} 
& No follow-up data & Follow-up data & PX2 or P Whitney U test \\
\hline N & 400 & 568 & 0.026 \\
Female, N (\%) & $222(56 \%)$ & $274(48 \%)$ & 0.037 \\
Age, mean \pm SD & $51 \pm 17$ & $53 \pm 16$ & 0.0014 \\
IGE & $90(23 \%)$ & $181(32 \%)$ & 0.0015 \\
Classic IGE & $51(13 \%)$ & $117(21 \%)$ & 0.404 \\
Abnormal anxiety & $27(7 \%)$ & $31(5 \%)$ & 0.306 \\
Abnormal depression & $21(5 \%)$ & $22(4 \%)$ & 0.105
\end{tabular}




\section{PBMC Immune-phenotyping}

Whole blood was collected following a protocol approved by the Ethics Committee of the University Hospital Leuven. Heparinized blood was rested for 2 hours at $18^{\circ} \mathrm{C}$ before extraction using ficoll separation protocol (LSM; MP Biomedicals, Belgium). PBMCs were frozen immediately in $10 \%$ Dimethylsulfoxide (DMSO, Sigma-Aldrich, Diegem, Belgium) $90 \%$ fetal bovine serum (FBS) at $-80^{\circ} \mathrm{C}$ for a maximum of 2 months.

Thawed cells were stained using antibodies (purchased from eBiosciences, Vienna, Austria, unless mentioned elsewise) against CD3 (SK7), CD4 (RPA-T4), CD8 $\alpha$ (RPA-T8), CD14 (61D3), CD19 (HIB19), CD25 (BC96), DC56 (MEM188), CD11c (3,9), CD123 (6H6), CD45Ra (HI100), CD31 (WM-59), CCR7 (3D12), HLADR (LN3), Va24Ja18 (6B11), CXCR5 (IgG23, R\&D) to identify CD4 T cell subsets (naïve, RTE, TEM, TCM, Th1, Th2, Th17, Tfh, IL-2-producing, Tregs), CD8+ T cell subsets (naïve, RTE, IFNYproducing, IL-2 producing), B cell subsets (Naive, switched, memory- B cells), $\gamma \delta$ T cells, DC, NK and NKT. Intracellular staining was performed for Foxp3 (206D, Biolegend, ImTec Diagnostics N.V., Antwerp, Belgium) using the eBioscience fix/perm buffers. Intracellular cytokine expression was measured following 5 hour stimulation at $37^{\circ} \mathrm{C}$ with Phorbol myristate acetate (PMA, $50 \mu \mathrm{g} / \mathrm{ml}$, Sigma-Aldrich) and lonomycin (500 ng/ml, Sigma-Aldrich) in RPMI1620 in the presence of Golgistop (1:250, from BD kit). Following stimulation, cells were treated with BD fixative and permeabilisation buffers and stained for IFNY (4S.B3), IL-4 (8D4-8), IL-17 (64DEC17) and IL-2 (MQ1-17H12). Acquisition was performed on a BD FACSCantoll with analysis by Flowjo (version 7.6.5, Ashland Or, USA).

\section{Cytokine analysis in supernatants of stimulated PBMCs}

Freshly isolated PBMCs were stimulated for 72 hour at $37^{\circ} \mathrm{C}$ and $5 \% \mathrm{CO} 2$ with $\mathrm{CD} 3 / \mathrm{CD} 28$ (SigmaAldrich). PBMC supernatant was collected and stored at $-80^{\circ} \mathrm{C}$ until analysis. Cytokine levels of IL13, IL8, IL1b, IL10 and TNF $\alpha$ were quantified by cytometric bead array (BD Bioscience, Erembodegem, Belgium). Samples were acquired on FACSCanto flow cytometer (BD Bioscience) and analysed by FCAP v3.0 analysis software (Soft Flow Inc., Pecs, Hungary).

\section{Cytokine analysis in plasma}

Plasma was prepared by centrifuging whole blood at $300 \mathrm{~g}$ for $15 \mathrm{~min}$ at $18^{\circ} \mathrm{C}$ and stored at $-80^{\circ} \mathrm{C}$ until assay. The levels of soluble IL-4, IL-6, IL-8, IL-10, IL-13, IL-17 and IFN $\gamma$ in thawed plasma was assessed by a Multi-spot assay system (V-plex kit: Cytokine and Proinflammatory Panel 1 Kits, Mesoscale Diagnostics) according to manufacturer's instruction (Meso Scale Discovery (MSD) Rockville, MD, USA). The outcome signals were read by Sector Imager 2400 (MSD). 


\section{Statistical analysis}

All statistical analyses of the clinical data were performed using SPSS, version 22 (IBM, SPSS software, Belgium). Continuous data were summarized by their mean and standard deviation. When serious deviations from normality were observed, medians and interquartile values were presented. For categorical variables, observed frequencies and percentages were presented. Comparisons between groups were made using a Pearson Fisher exact or Chi-square test (reported as px2) when appropriate. Continuous variables such as age in relation to binary variables (IGE, PI-IBS) were analyzed by binary logistic regression analysis (reported as pLR). Variables identified to be associated with risk IGE or PI-IBS in univariate analyses were included in the multivariable logistic model. As simulation models have shown that regression coefficients and other indicators of risk may be unreliable if fewer than 10 events are included in the models, a ratio of 10 IBS cases per predictor variable was required to ensure model stability (2). Beta regression coefficients, odds ratios, and their corresponding $95 \%$ confidence interval $(\mathrm{Cl})$ for the multivariate model were reported with the criterion for statistical significance set a priori at 0.05 . Multicollinearity was considered to be significant if the variance inflation factor exceeded 10.

To test the relative contribution of psychological status at the moment of the outbreak (anxiety, depression, somatization) and development of IGE to the risk of developing IBS at follow-up in general, and, more specifically, test whether the effect of psychological variables on the risk of developing IBS at follow-up is direct or indirect through an increased risk of development of IGE, mediation analysis was performed (3) using SAS 9.4 software (SAS Institute, Cary, NC, USA). Mediation analyses requires estimating three regression equations (3): 1) (logistic) regression of the psychological variable (independent variable) on IBS status at follow-up (dependent variable); 2) (logistic) regression of the psychological variable (independent variable) on IGE status (mediator) and 3) (logistic) regression of both the psychological variable (independent variable) and IGE status (mediator) on IBS status at follow-up (dependent variable). The effect of the psychological variable on IBS status at follow-up would be totally mediated through IGE status if adding IGE (in model 3) renders the (significant) effect of the psychological variable on IBS status (from model 1) nonsignificant.

All statistical analyses of the samples were performed using Graphpad Prism (Prism 6, version 6.02, Graphpad Software, CA, USA). Normality of the data was checked by D's Agostino and Pearson omnibus normality test. Non-parametric data were compared by Man-Whitney test or the KruskalWallis test followed by Dunn's correction for multiple comparisons when appropriate. Significant values were set at $p<0.05$. For linear correlation, $p$ and $R 2$ values were calculated using Pearson correlation test. 
1

2

3

4

5

6

7

8

9

10

11

12

13

14

15

16

17

18

19

20

21

22

23

24

25

26

27

28

29

30

31

32

33

34

35

36

37

38

39

40

41

42

43

44

45

46

47

48

49

50

51

52

53

54

55

56

57

58

59

60

Non-metric multidimensional scaling was used to visualize dissimilarities of samples between treatments. The ordination was created in R 3.1.0 using R-package vegan with custom scripts. Distance matrix was calculated with Bray-Curtis dissimilarity index on original data. To test for the contribution of each variable to the resulting distance matrix, we applied Mantel test in which the original distance matrix was compared with a temporary distance matrix where one variable (cell subset) was removed at a time. The test was run with 999 permutations. 


\section{Supplementary figure legends:}

sFig. 1. Serum cytokine levels in acute infectious phase

Serum cytokine levels were assessed in the acute infectious phase in subject who will become symptom free (IGE+ IBS-) or will develop PI-IBS (IGE+ IBS+) at 1 year follow-up. Cytokine levels were not different when comparing subjects suffering from acute gastroenteritis with healthy volunteers (HV). Moreover, there was no difference in serum cytokine level when comparing subjects who will develop IBS compared to those who will not.

sFig. 2. Analysis of gastrointestinal symptoms in post-infectious and new-onset IBS patients compared to healthy individuals, 1 year after the outbreak

Mean GSRS score per question (1-15) and total GSRS score (SUM) are presented on $X$ axis for postinfected no symptoms group (IGE+IBS-), post-infectious IBS (IGE+IBS+), the exposed group (IGE-IBS-) and the new-onset IBS group (IGE-IBS+). ${ }^{*} p<0.05 ;{ }^{* *} p<0.001 ;{ }^{* * *} p<0.0001$ Kruskal Wallis test with Dunn's multiple testing correction

sFig. 3. PBMC phenotyping analysis 1 year follow-up

Phenotypes of immune cells ( $B$ cells and subgroups, DC cells and subgroups and T cells) are not different between PI-IBS (IGE+IBS+), new onset IBS (IGE-IBS+), and their respective control groups (IGE+IBS- and IGE-IBS-).

\section{sFig. 4. Plasma cytokine analysis 1 year follow-up}

One year after the outbreak, cytokine levels are assessed in plasma. There are no differences in plasma cytokine levels for IL17, IFNg, IL10, IL4, IL6, IL8 or IL13 when comparing PI-IBS (IGE+IBS+), new onset IBS (IGE-IBS+), and their respective control groups (IGE+IBS- and IGE-IBS-).

\section{sFig. 5. One year follow-up inflammatory mRNA expression in rectal biopsies}

One year after the outbreak, mRNA expression levels of inflammatory genes were assessed in rectal biopsies. There are no differences in inflammatory gene expression levels in rectal biopsies when comparing PI-IBS (IGE+IBS+), new onset IBS (IGE-IBS+), and their respective control groups (IGE+IBSand IGE-IBS-). 
(1) Pfaffl MW. A new mathematical model for relative quantification in real-time RT-PCR. Nucleic Acids Res 2001;29(9):e45.

(2) Concato J, Feinstein AR. Monte Carlo methods in clinical research: applications in multivariable analysis. J Investig Med 1997;45(6):394-400.

(3) Baron RM, Kenny DA. The moderator-mediator variable distinction in social psychological research: conceptual, strategic, and statistical considerations. J Pers Soc Psychol 1986;51(6):1173-82. 

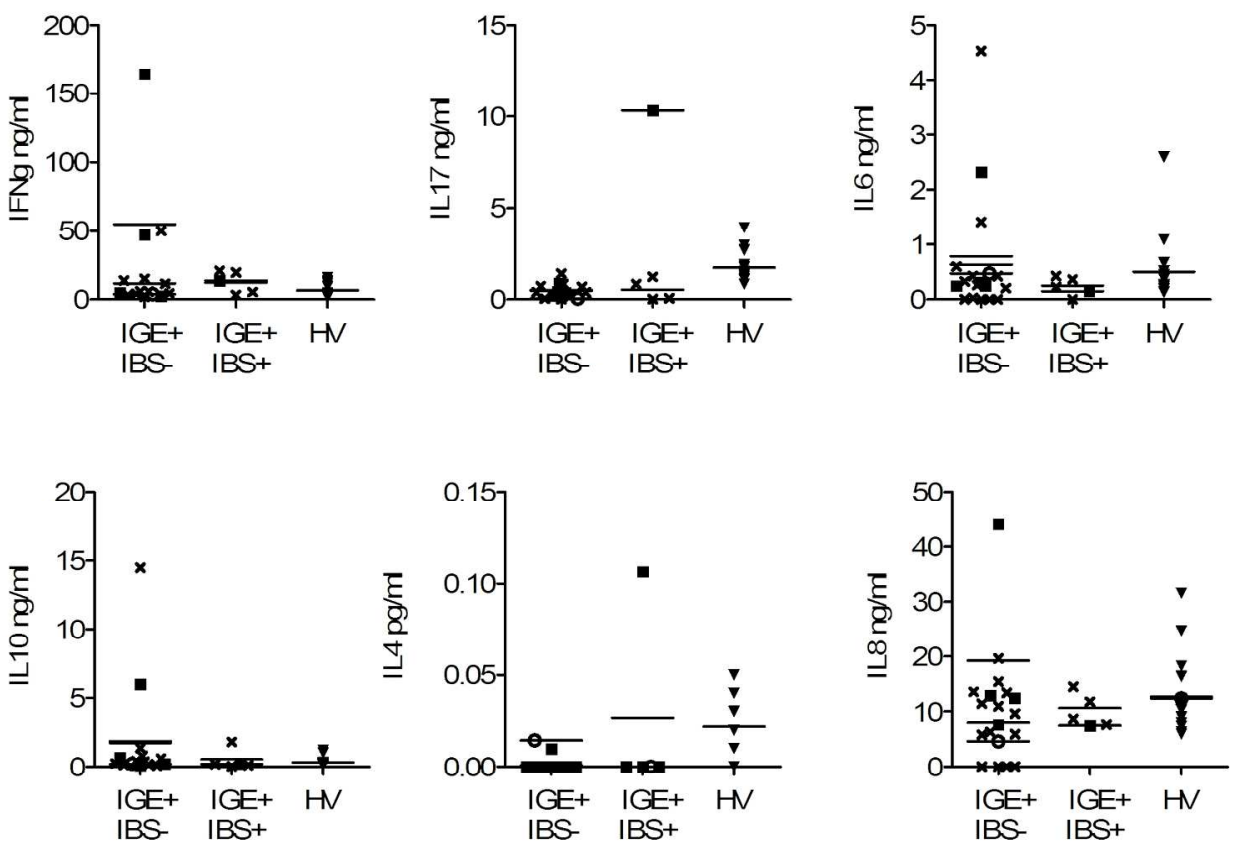

$$
\times
$$

classic

- moderate

- IGE wo diarrhea

Serum cytokine levels in acute infectious phase

Serum cytokine levels were assessed in the acute infectious phase in subject who will become symptom free (IGE+ IBS-) or will develop PI-IBS (IGE+ IBS+) at 1 year follow-up. Cytokine levels were not different when comparing subjects suffering from acute gastroenteritis with healthy volunteers (HV). Moreover, there was no difference in serum cytokine level when comparing subjects who will develop IBS compared to those who will not.

$199 \times 166 \mathrm{~mm}(300 \times 300 \mathrm{DPI})$ 
Analysis of gastrointestinal symptoms in post-infectious and new-onset IBS patients compared to healthy individuals, 1 year after the outbreak

\section{Mean GSRS score per question (1-15) and total GSRS score (SUM) are presented on X axis for post-infected no symptoms group (IGE+IBS-), post-infectious IBS (IGE+IBS+), the exposed group (IGE-IBS-) and the new-onset IBS group (IGE-IBS+). * $\mathrm{p}<0.05 ; * * \mathrm{p}<0.001 ; * * * \mathrm{p}<0.0001$ Kruskal Wallis test with Dunn's multiple testing correction}

$195 \times 160 \mathrm{~mm}(300 \times 300 \mathrm{DPI})$ 

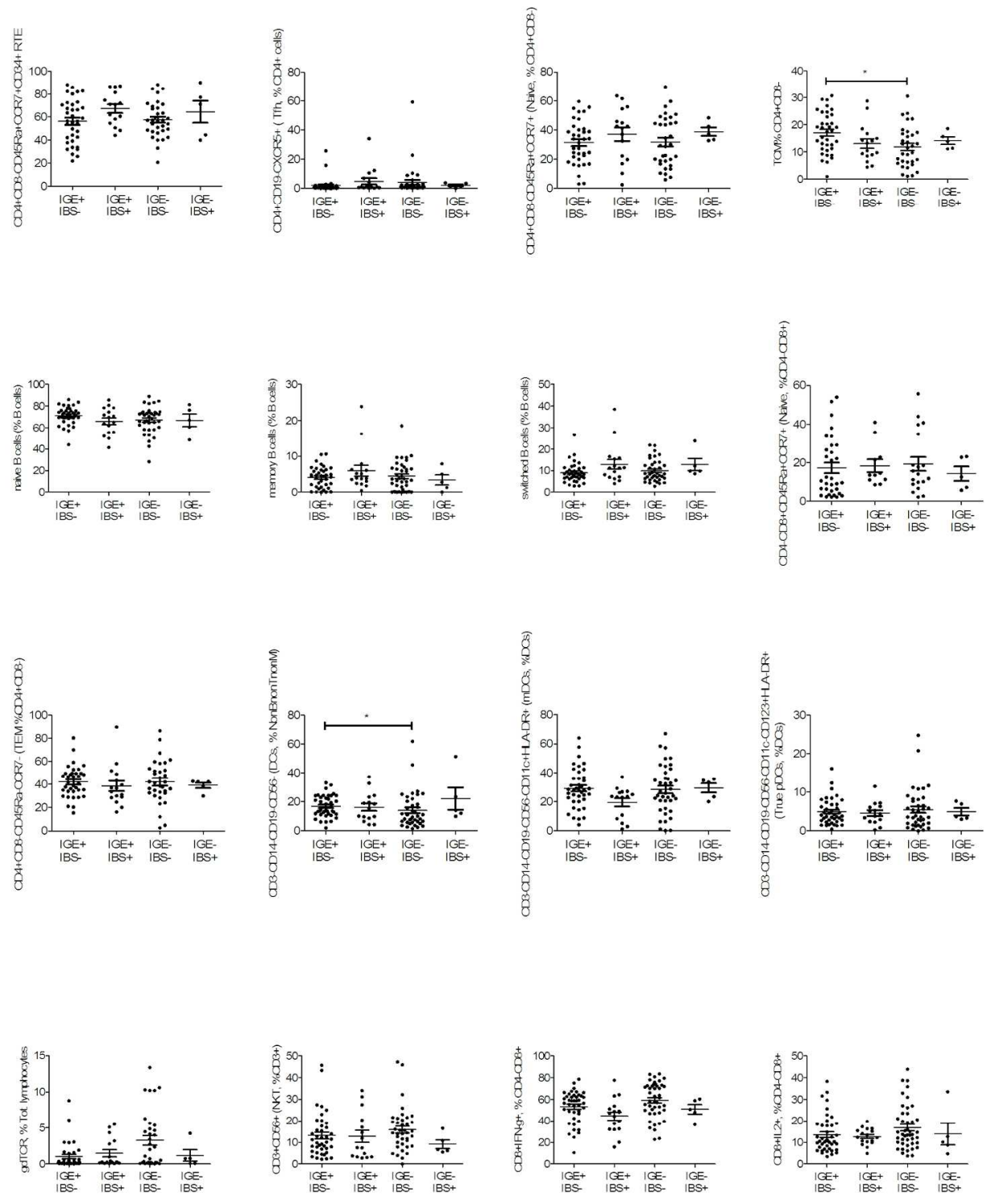

PBMC phenotyping analysis 1 year follow-up

Phenotypes of immune cells ( $B$ cells and subgroups, DC cells and subgroups and T cells) are not different between PI-IBS (IGE+IBS+), new onset IBS (IGE-IBS+), and their respective control groups (IGE+IBS- and IGE-IBS-).

$180 \times 221 \mathrm{~mm}(300 \times 300 \mathrm{DPI})$ 

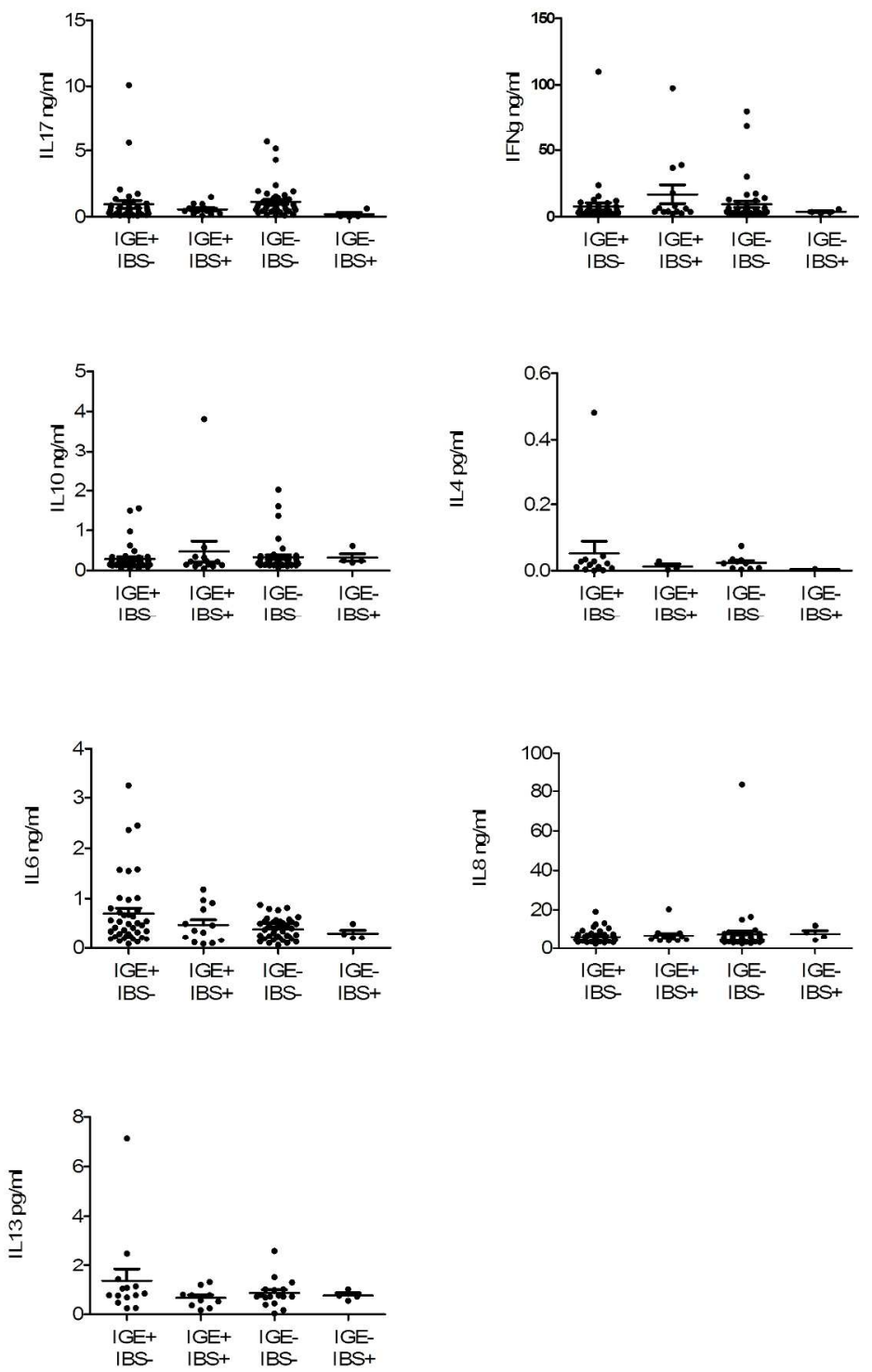

Plasma cytokine analysis 1 year follow-up

One year after the outbreak, cytokine levels are assessed in plasma. There are no differences in plasma cytokine levels for IL17, IFNg, IL10, IL4, IL6, IL8 or IL13 when comparing PI-IBS (IGE+IBS+), new onset IBS (IGE-IBS+), and their respective control groups (IGE+IBS- and IGE-IBS-).

$$
177 \times 281 \mathrm{~mm}(300 \times 300 \text { DPI) }
$$



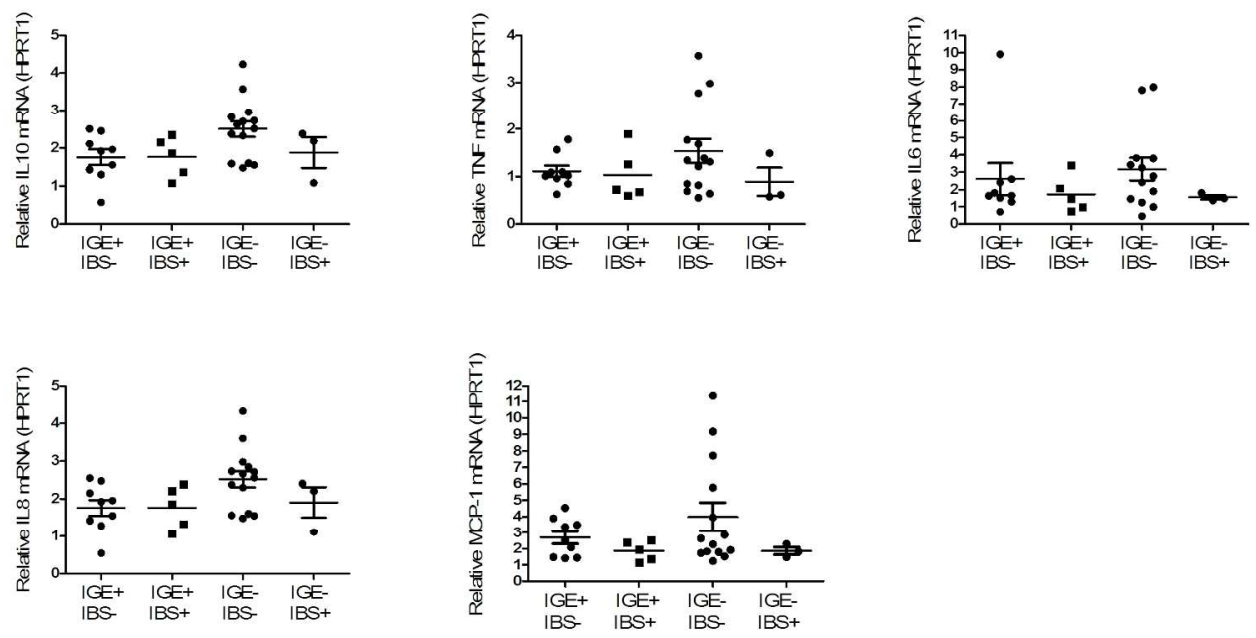

One year follow-up inflammatory mRNA expression in rectal biopsies

One year after the outbreak, mRNA expression levels of inflammatory genes were assessed in rectal biopsies. There are no differences in inflammatory gene expression levels in rectal biopsies when comparing PI-IBS (IGE+IBS+), new onset IBS (IGE-IBS+), and their respective control groups (IGE+IBS- and IGEIBS-).

$212 \times 113 \mathrm{~mm}(300 \times 300 \mathrm{DPI})$ 